$\gamma$

A major purpose of the Technical Information Center is to provide the broadest dissemination possible of information contained in DOE's Research and Development Reports to business, industry, the academic community, and federal, state and local governments.

Although a small portion of this report is not reproducible, it is being made available to expedite the availability of information on the research discussed herein.

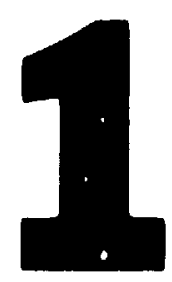




\section{LA-UR $85-3 \$ 40$}

$\cos 15.50120120-2$

$001: 1 i^{2} 1985$

TITLE LAMPF - ITS ORIGINS, HISTORY, AND ACCOMPLISHMENTS

AUTHOR(S): LOU18 Rosen

LA-UR $-85-3340$

DE86 000814

SUBMITTED TO

Megon 50, The Jubilee of the Megon Theory, Kyoto, Japan, 15-17 August 1985

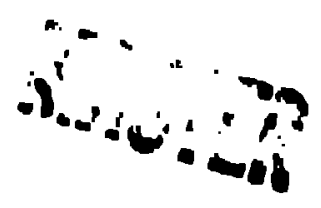

\section{DISC:LAIMER}

This repon we preparod a an eccount of work opemaured by an enonicy of the IIniled Sintew (jowernment. Nather the Uniled Shates fluwernment mor any aconsy thereof, mur any of thair employees, makes any warranty. exprese or implied, of anumes any legel liability or respunni.

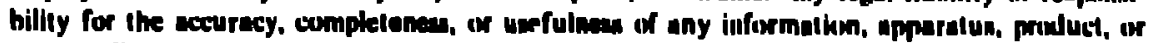

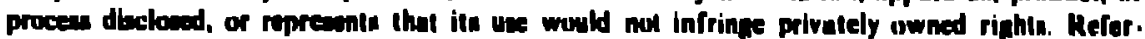

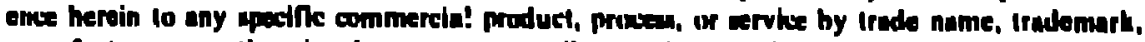

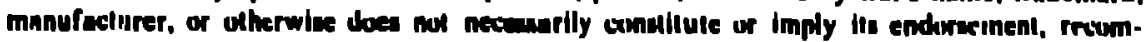
mendation, of favorias by the United Sinter (hovernment in any agency thereal. The viewn

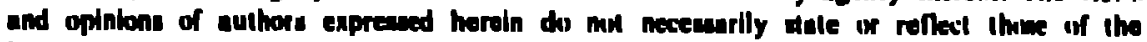
Uniled shale (lovernmont of any apency therear. 


\title{
LAMPF-ITS ORIGINS, HISTORY, AND ACCOMPLISHMENTS
}

by

\author{
Louis Rosen
}

\section{INTRODUCTION}

The time was a quarter of a century ago. The science of nuclear physics was not even 50 years old, but it had already provided fantagtic options for the entire world. It promised a vehicle for a high level of energy production fa. into the future and it dramatically altered the role of science and technology in defense, industry, medicine, and even agriculture. It had been responsible for ending a great war and for placing civilization in hostage against another international conflagration. But it also challenged the determination, wisdom, and ingenuity of the world's political leadership to find ways, while there is still time, for the nations of the world to live at peace. Los Alamus, under the able leadership of Norris Bradbury, was seeking way 9 to diversify its cortributions to the nation while enhancing its vis'ility and vitality as one of the nation's foremost national security laboratories.

In 1960, the foundation of nuclear physics stnod mainly on quantum theory, Yukawa theory, relativity, phenomenological models, and on the premise that the nucleus, its structure, and its properties could be described-in some circumstances as a collection of nucleons, each inoving more or less independently of all the others in a mean field created by all nucleons in the nucleus, and in other circumstances as simply a collection of strongly interacting nucleons, a liquid drop. In either case, nucleonic degrees of freedom were the prime factor in nuclear statics and nuclear dynamics.

Professor Yukawa's pion had been discovered. Many of its properties had been unveiled and they agreed with the Yukawa theory. There were, however, puzzles in the behavior of nuclei and nuclear reactions. It was postulatnd that pionic degrees of freedom must be a part of any comprehensive description of nuclei and nuclear reactions. Experiments with pions had been carried out, but the intensity and quality of the beams were completely inadequate to launch the studies befitting the particle that was heralded as the messenger of the strong nuclear force, analogous to the photon in quantum electrodynamics (QED) that was already well established as the quantum of the electromagnetic field responsible for communication between rlectrons.

It was in this climate, as progress in classical nuclear physics relative to the effort being invested was already slowing, that the idea for meson factories was conceived and began to germinate.

It was also in this climate that a new regime of physics intermediate-energy physics way born, with the hope and promise that it would rojuvenate nuclear science and bridge the gap between nuclear and aubnuclea: (high-energy) physics.

\section{THE MESON FACTORIES}

The main vehicle for keeping thi promise, and bringing the pion to bear on a vast array of problems, has been the meson factories. 'loday there exist such facilities in Switzerland (SIN), in Canada (TRIUMF), and in ule USA (LAMPF). A fourth is just now being 
completed in the Soviet Union. They are enormous enterprises-the current replacement value of LAMPF is $\$ 350$ million, not including the part devoted to national security problems. But they accommodate hundreds of scientists from around the world and by so doing generate political as well as intellectual and economic capital. Proton facilities, together with heavy-ion accelerators and electron facilities, form a triad on which stands the present edifice of experimental nuclear science. Each leg of this triad is dependent, to a greater or lesser extent, on the other two. However, in terms of versatility, the size of the community it serves, and the relatively short-term application of the knowledge base and people base for which nuclear science is responsible, the meson factory part of the above triad is by no means the least important comporent. I discuss this component from the standpoint of the facility I know best, namely LAMPF.

\section{THE EVOLUTION OF LAMPF}

The first document proposing LAMPF was written in 1962. There followed 6 years of political trials and tribulations, but also of great technical progress. October 1968 brought the first funds for construction of the facility. Within 4 years, on budget and on time, the first full-energy beam was observed in the switchyard. This was, indeed, a joyous orcasion, as you can see from Fig. 1. We had exceeded the energy for proton linacs by a factor of 4 , but we were still far from achieving our geal of an external beam 1000 to 10000 times more intense than any then available, a feat most experts had warned was not achievable-but they were wrong.

LAMPF as it exists today is shown in Fig. 2. In the foreground are the experimental areas-the pion-muon area, the Nucleon Physics Laboratory, the High-Resolution Proton Spectrometer, the Biomedical channel, the Neutrino areas, and the Control Room. The accelerator is underground for shielding; in the background are the Laboratory-Office Building and the Equipment Test Laboratory. Looking to the west we see the mountain range that cradles the Valle Grande, the largest extinct volcano in the northern hemisphere. We hope it remains extinct.

On: che south side of the highway is the national security facility, the Weapons Neutron Research/Proton Storage Ring (WNR/PSR) complex. The thrust here is the study of condensed matter physics, nuclear physics, biology, and national security probiems-all with pulsed neutrons. With the PSR in operation, we will have a world-class facility, as good as any in existence or under construction, for neutron time-of-flight studics.

A schematic of the area downstream of the switchyard is shown in Fig. 3.

Figure 4 shows the installation of a new neutrino experiment ${ }^{1}$ that will focus on neutrino oscillations, to which 1 shall return.

\section{INVOLVEMENT OF THE SCIENCE COMMUNITY}

$A$ key, and in retrospect very wise, policy derizion was made at the very beginning to decply involve the scientific community in the development and governance of LAMPF. The basic mechanism for achieving this, which hes withatood the test of time, is shown in fig. 5. The scientific community is represented by the $\sim 800$-member LAMPF Users (iroul, n nonproflt corporation constituted under the laws of New Mexico. 
The heart of tine advisory mechanism for determining experimental priorities is the Program Advisory Committee (PAC). Appointed by the Director of LAMPF from nominations made by the Board of Directors (BOD), who are elected by the Users Group, the PAC is composed of 24 highly respected scientists appointed for staggered 3-year terms. They meet twice each year for about 1 week, during which time all active and proposed experiments are reviewed and ranked according to the physics to be learned, technical feasibility, and perceived competence of the investigators. These criteria are, of course; to some extent subjective. However, one-third of the PAC and one-half of the BOD are refiaced each year; this tends to prevent a provincial view of the worth and feasibility of proposed experiments.

The BOD also appoints a Technical Advisory Panel (TAF), which studies and makes recommendations for new capabilities. After review by the BOD, which sometimes convenes additional advisory conmittees, a decision is made on whether to recommend implementation of a given improvement proposal. This process often involves the BOD in the LAMPF budget, to which they have full access. When, therefore, a decision is made by the management of LAMPF for a major new capabinity, it can count on the support of a large part of the user community. This support is vital.

\section{PROMISES vS PERFORMANCE}

Figure 6 shows a comparison of what we promised with what has been achieved in accelerator and experimental capabilities. The key promise was high intensity. It was achieved. In fact, LAMPF today generates a proton beam intensity that still exceeds the combined beam intensities of ali the world's proton accelerators operating at energies above the pion-production threshold.

An area where we have made sorre of our most visible accomplishments, none of them promised, is in technology transfer to the private and public sectors. I shall return to this facet of our activities.

Annther major promise was that L $\Lambda$ MPF would serve a large community of scientists. In any yefur, approximately 350 scientist 3 from about 90 institutions work at LAMPF. To date, approximately 1100 papers have been published in refereed journals and more than 100 doctoral theses have been completed.

Figure 7 lists the various naticns represented at LAMPF during the past 2 years.

\section{RECENT SCIENTIFIC GOALS AND ACHIEVEMENTS}

Just as a quarter of a century ago there svas good reason to believe that pionic degrees of freedon ure essential to a description of nuclear structure and nuclear reactions, so does it now appear that quark and gluon degrees of freedom may be essential components of a deeper understanding of nuclear phenomena.

To my mind, the most important open questions in nurlear science, as a whole, revolve about whether quantum chromodynamics (QCD), involving quark and gluon degrees of freedom, can fully describe strong interactions. We therefore now face the question of how to explore the consequences of QCD at energies where quark confinement is operative. To do this will probably require a large variety of experiments of high accuracy. 
Correspondingly, regarding the weak interaction and its unification with the electromagnetic interaction, we now must ask how we can further validate and extend the standard model of electroweak interactions.

Other major goals are to determine the limits of symmetries and conservation laws and to extend our knowledge of QED by testing its limits.

Experiments at LAMPF' are therefore focusing on many areas (Fig. 8) and include the research goals listed in Fig. 9. I shall touch on some of these areas.

\section{A. The Strong Interaction}

Notwithstanding a world-wide monumental effort to determine, unambigususly, the scattering amplitudes for the free nucleon-nucleon interaction in the reginn up to $800 \mathrm{MeV}$, this problem is not yet solved. Although the p-p scattering amplitudes are now reliably determined, that is not the case for $n$-p scattering. However, high-intensity polarized-proton sources seem to be within reach. The next 6 years should see this problem eliminated. It will then become more fruitful to enhance our attack on the spectroscopy of nuclei at high excitations and also on matter distributions in complex nuclei.

But even with presently available polarized-proton intensities, dramatic results have emerged from nucleon-nucleon and proton-nucleus elastic-scattering and polarization experiments. Professor Masaike and his group have recently participated in a milestone experiment ${ }^{2}$ that measured the spin observables in the scattering of polarized protons by a polarized-protor target at very small scattering angles-in the region of the Coulombnuclear interference. This is important for understanding pion-nucleus interactions.

In Fig. 10 we see proton scattering ${ }^{3}$ from ${ }^{208} \mathrm{~Pb}$. The solid curve is a thec atical prediction based on the non relativistic Schrödinger equation. The disagreement seems irreconcilable because it now appears that cross sections at large angles, periodicity, and the spin observables are not correctly predicted by the conventional theoretical description. It is, however, becorning apparent that the coupling to negative-energy degrees of freedom, implicit in relativistic Dirac representations, can lead to impressive advances in the ability of theory to reproduce the data, including all the spin observables.

The view that relativistic effects must be included in a description of low-energy nuclear phenomera is attracting many adnerents and may turn out to be one of the most important discoveries in nuclear science during the past decade. Figure 11 shows polarized-proton scattering from ${ }^{17} \mathrm{Ca}$ along with theoretical predictions for the spinrotation parameter. ${ }^{5}$ May I reinind you that the spin-rotation parameter $Q$ is a function of two spin observables and is highly sensitive to the scattering amplitudes and phases. The solid curve, the prediction of a phenomenological model of proton-nucleus elastic scatterinz, is based on the Dirac equation; the broken curve is baseci on the Schrödinger equation. Figure 12 shows the apparatus used to obtain these data. The spectrometer has a resolution of better than $50 \mathrm{keV}$ at $800 \mathrm{MeV}$.

The overall advances in our understanding of the proton-nucleus interaction have also rekindled the long-held hope that these advances, together with $\pi^{ \pm}$-nucleus scattering, muonic atoms, and electron scattering, will allow determination of detailed neutron density form factors.

The above developments have resulted from experiments of high accuracy. They were made nossible by high-quality, high-intensity beams, by advances in polarized beams 
and polarized targets, and by state-of-the-art instrumentation, especially spectrometers, detectors, and computerized data acquisition.

Data obtained during the past several years are fueling attempts to extend the scope of nuclear science to include quark-gluon degrees of freedorn. This appears to be the next logical step in the evolution of the field and is a major stimulus for higher energy meson factories, the kaon factories. But some progress may be possible with current capabilities. A recent example is an experiment conducted by T. A. Carey et al. ${ }^{6}$

They take note of the European Muon Collaboration (EMC) experiment, ${ }^{7}$ which was designed to test quantum chromodynamics, a theory which postulates that quarks interact through the exchange of gluons much like quantum electrodynamics (QED) postulates that electrons interact through the exchange of photons. Neither quarks nor gluons have been seen in a free state, but they have attracted many believers.

The EMC experiment, Fig. 13, measured the electromagnetic structure function $\left[F^{2}\left(\chi, Q^{2}\right)\right]$ for deep inelastic muon scattering from free nucleons (deuterium) and from iron nuclei for $E_{\mu} \sim 200 \mathrm{GeV}$. The experiment was designed to probe the quark distribution in the same way that quasi-elastic electron scattering probes the charge distribution in a nucleus. For short wavelengths the structure function of a heavy nucleus was expected to be the incoherent summation over the structure function of the constituent nucleors. But this did not turn out to be so. A tempting explanation involves the communication of quarks in one nucleon with quarks in other nucleons--the distribution of quarks then depends upon the nuclear environment.

However, Llewellyn-Smith and Ericson and Thomas ${ }^{8}$ explann the EMC observation in terms of classical nuclear physics by invoking the enhancement of the pion. field in a heavy nucleus as a result of the attractive nature of the pion-nucleon interaction. The extra pions, the so-called pion condensate, are then the source of the additional quarks, and our conventional view remains intact.

The recent LAMPF experiment looked for the extra pions. Figure 13 pictures the situation as envisioned by T. A. Carey and his colleagues.

Whereas the electromagnetic field does not permit the proper coupling to pions, thus excluding the use of electrons, polarized protors do offer a proper coupling and an adequate sensitivity. The key is to measure a complete set of polarization transfer observables in two scattering experiments. As in the EMC experiment, one target consists of free nucleons (deuterium) and the other is a complex nucleus (lead). By taiking the ratios of the longitudinal itsponses for deuterium and lead to the transverse responses for the same targets, the experimenters isolate the pionic coupling of the proton to the nucleus.

Figure 14 illustrates the preliminary results from this investigation.

The measurenent of appropriate polarization transfer observables for $(\vec{p}, \vec{p})$ ) showed no differcaces between lead and deuterium and no enhancement in the nuclear pionic field. Thus the initial interpretation of the EMC experiment has not been negaled. These results support the inclusion of quark degrees of freedom in ruclear theories.

Another signature of quark presence in nuclei was also explored at LAMPF. It involves single and double pion charge exchange.

Figure 15 shows the $0^{\circ}$ differential cross section for the isobaric-analog-state (IAS) transition in the reaction ${ }^{0}{ }^{11} \mathrm{C}\left(\pi^{+}, \pi^{0}\right)^{14} \mathrm{~N}$. The excitation function goes through a deep 
minimum near $50 \mathrm{MeV}$. This minimum is a reflection of the $s$ - and p-wave interference in the elementary pion-nucleon interaction. It was suggested by the calculations of Gerry Miller that a comparison of the pion double-charge-exchange data with the single-chargeexchange data might yield evidence for six-quark cluster contributions. Miller predicted that the $50-\mathrm{MeV}$ :ross section at $0^{\circ}$ for the double-isobaric-analog-state (DIAS) transition would be about $10 \mu \mathrm{b} / \mathrm{sr}$ at the same time that the IAS cross section would be $\$ 1 \mu \mathrm{b} / \mathrm{sr}$. This is unusual, because the typical situation at higher energies is that IAS cross sections are several microbarns per steradian and DIAS cross sections are $\$ 1 \mu \mathrm{b} / \mathrm{sr}$.

Figure 16 displays the ${ }^{14} \mathrm{C}\left(\pi^{+}, \pi^{-}\right)^{14} \mathrm{O}$ cross sections, as a function of angle, and compares them with the predictions of the six-quark mode!. ${ }^{10}$ The data are by no means conclusive, but they may suggest a contribution from six-quark clusters. The forward-angle peaking of the anguiar distribution had not been expected frum earlier optical-poiential calculations.

It has been long recosnized that the pion can play a special role in sorting out nucleonparticle and -hole states. Near the $\Delta$ :esonance, the ratio of the cross section for

$$
\frac{\pi^{+} p}{\pi^{+} n} \text { and } \frac{\pi^{-} n}{\pi^{-} p} \text { is } \sim 9 \text {, }
$$

causing large differences in the scattering of $\pi^{+}$and $\pi^{-}$when single-particle proton and neutron states are excited. The resulting spectra can be used to determine the degree of isospin mixing ${ }^{11}$ (see Fig. 17).

A diamatic manifestation of the usefulness of the pion as a nuclear probe is seen in the excitation of collective states by way of pion single and double charge exchanges. Here the spin-isospin properties of pions make them unique among nuclear probes.

In single-charge-exchange rtactions $\left(\pi^{ \pm}, \pi^{0}\right)$, the $\pi^{0}$ is observed by virtue of its decay into two gamma rays. A special $\pi^{0}$ spectrometer ${ }^{12}$ (Figs. 18 and 19) was developed for this purpose. Data obtained ${ }^{13}$ (Fig. 20) led to the first identification of the giart isovector-monopole resonance (IVMR), a collective motion in which lixe protons and neutrons oscillaie out of phase along the nuclear radius (the protons breathe in and out while the neutrons breathe out and in). The exchange reaction $\left(\pi^{-}, \pi^{0}\right)$ is ideally suited for exciting the IVMR. Because the pion has zero spin, excitation of spin-flip states at forward angles is strorgly inhibited; thus mainly isovector states are excited. Data, sucin as those shown in Fig. 20, established the IVMR as a - stematic feature of complex nuclei. In addition to constraining the isovector componen, of the interaction of nucleons in nuclei, such data may provide ins'ght into the compressibility of nuclear matter, which has important implications for astruphysics.

We are finally beginning to understand the role of the $\Delta$ in the nuclear mediumespecially how it propagates and how it irfluences the excitation of specific levels.

The main point is that $\pi^{ \pm}$-nucleus reactions are highly selective in terms of the statcs they excite, thus pernitting investigation of reaction mechanism properties and nuclearstructure effects. However, systematic studies are required over wide ranges of energies and nuclei.

The original hope that high-intensity, good-quality beams of pions can probe nuclei in ways not otherwise feasible is being realized. Pions are now a recognized tool in the study of the manifestations and mechanisms by which nuclei absorb and emit energy. 
Observation of the $(\pi, \eta)$ Reaction on Nuclei. The $\eta$ meson, like the pion, is a nonstrange pseudoscalar meson classified in the same $\mathrm{SU}(3)$ octet as the pion.

The $\pi^{ \pm}, \eta$ reaction is analogous to the $\pi^{ \pm}, \pi^{0}$ reaction except that it involves two particles belonging to different isospin multiplets and the large mass difference requires a large mcmentum transfer. This reaction has been recently observed very near threshold. Figure 21 shows how this was done. ${ }^{4}$

The S1 scintillator counts the pions and determines zero time for the neutron time of flight. the BGO counters measure the coincident garnma rays emitted from $\eta \sim 2 \gamma$ decay, and four sets of scintillation counters detect neutrons from $\pi^{-}+p \rightarrow \eta+n$. Events accompanied by charged particle emission are vetoed. The $\eta$ production was observed from protons and from ${ }^{12} \mathrm{C}$.

Figure 22 shows the time-of-flight spectrum. The first peak is from $\pi^{0}$ decay produced by $\pi^{-}+p \rightarrow \pi^{0}+n$, the next peak comes from the neutrons in ihis reaction, and the two remaining peaks are from the $\pi^{-}+p \rightarrow n+\eta$, corresponding to neutrons produced at forward and backward angles in the center-of-mass system. The cross section for $\pi^{ \pm}+p \rightarrow$ $n+\eta$ is $83 \mu \mathrm{b} / \mathrm{sr}$ at $\theta_{\text {c.m. }}(\eta)=144^{\circ}$ and $86 \mu \mathrm{b} / \mathrm{sr}$ at $\theta_{\text {c.m. }}(\eta)=25^{\circ}$. The challenge now is to investigate the interaction of $\eta$ mesons with the nuclear mediun.

None of the above examples directly reflects the primary philosophical impact of the meson factories on nuclear physics-namely, the transition from classical nuclear physics, in which only structureless nucleons and the two-body potentials between them play the decisive role, to the present richness of tise field. Many more degrees of freedom play a role in nuclear physics. These additional degrees of freedom are represented by the mesons, nucleonic excitations, and antinucleons, and their effects show up even below pion-production thresholds. Because of the copious data generated by the meson factories, we : e learning quantitatively how these additional hadrons behave in the nuclear medium ano she role they play in nuclear physics. Such an evolutionary way of looking at nuclear phy sics appears to be an intermediate but necessary step toward the even greater richness implied by a parton (quark) spectroscopy. This exciting beginning will no doubt be carried further by the higher energy $\mathrm{cw}$ electron accelerators and of course by the 3o-calied kaon factories.

The above strong-interaction program is augmented by nuclear chemistry, exploring reaction mechanisms and nuclear dynamics. The program also includes the production and study of nuciei far from the line of stability and a comparison of results with theoretical predictions.

\section{B. Atomic: Physics}

Spectroscopy of $\mathrm{H}^{-}$. Meson factories have proved to be remarkably versatile for attacking diverse problems in powerful ways, often providing the only fersible means ior confronting an important puzzle.

Such a lor.-standing problem is $\mathrm{H}^{-}$ion spectroscopy. Conventional means are in. adequate to measure (1) the resonance structure in single photodetachment and (2) the dcuble photodetachment of $\mathrm{H}^{-}$electrons. (There are as yet no tunable vacuum-ultraviolet lasers.) Although nothing more than basic quantum mechanics is involved and the forces are known, we still have the complexity of a three-body problem. Its iull comprehension lies ahead. LAMPF permits exploration of this physics by way of collidings beams. ${ }^{15}$ We do 
it with mirrors, using the Doppler effect (Fig. 23). A laser beam of appropriate energy and variable direction intersects the $800-\mathrm{MeV} \mathrm{H}^{-}$beam. Introduction of a modest magnetic field permits the $\mathrm{K}^{-}$ion to be studied in electric fields of about $5 \mathrm{MV} / \mathrm{cm}$. The results (Fig. 24) are as elegant as the equipment is simple. Center-of-mass energies cover precisely the range of interest, 0.5 to $20 \mathrm{eV}$, with resolution in the milli-electron-volt region.

The work on the spectroscopy of the $\mathrm{H}^{-}$atom turns out tu have dramatic practical consequences. One of the major options in the Strategic Defense Initiative could be neutral particle beams. Such beams can only be produced by accelerating and focusing $\mathrm{H}^{-}$ ions. Beam emittance and beam position are primary considerations and are intimately connected with the photodetachment process. Furthermore, the experimental technology developed to explore the photodetachment process is precisely applicable to the studies that must now be made to evaluate the potential of neutral particle beams.

\section{Electromagnetic and Weak Interactions}

Tests of Quantum Electrodynamics (QED). The Stopped-Muon Channel at LAMPF provides a copious source of polarized low-energy muons, essentially free of pions and reasonably free of electrons. Highly sophisticited experiments have been mounted by the Yale group and their collaborators, resulting in greatly improved knowledge of the spectroscopy of muonium and, as a by-product, improved values of the fine-structure constant and the ratio of muon-to-proton magnetic moments.

Muonium had already been discovered by Professor Vernon Hughes and his group at Yale when LAMPF was being designed. It has since become a powerful tool for determining the limits of an astoundingly accurate field theory, QED. This has come about through precise measurements of the hyperfine structure interval and the Zeeman effect.

Why is muonium an ideal system for determining the limits of QED? Because it comprises two different dimensionless and structureless lejtons, free of any known stronginteraction effects. Muonium can also serve as a laboratory for exploring unknown weakor strong-interaction effects in the muon-electron system.

Discovery of a means to produce muonium in vacuurn has led to the observation of the Lamb shift in muonium ${ }^{16}$ and also to the possibility of new kinds of experiments, such as the conversion of muonium to antimuonium. The recent detection, at TRIUMF and LAMPF, of the $2 S$ state in muonium ${ }^{17}$ and the observation of the rf-induced transition $2 S_{1 / 2} \rightarrow 2 P_{1 / 2}$ at the rate of several per hour open the door to another milestone test of QED. This measurement of the Lamb shift does not involve effects of proton stiucture that introduce difficulties in the interpretiation of the Lamb shift in hydrogen. Similar experiments have been performed at other meson factories.

Another test of QED carried out at LAMPF involved the Dalitz decay of neutral pions: $\pi^{0}$ is produced from $\pi^{-} p \rightarrow \pi^{0} n$, and the decay rate is measured for $\pi^{0} \rightarrow e^{+} e^{-\gamma}$ relative to $\pi^{p} \rightarrow \gamma \gamma$. The ratio is sensitive to vacuum polarization contributions: the results obtained are consistent with QED predictions to a high level of precision. ${ }^{18}$

Muonic Atoms. Muorss are useful in many other ways. The initial conjecture by Bethe, that both muonic-atom and electron-scattering data would be required to determine charge distributions of nuclei, proved correct. The point is that, with negative muons, one can form hydrogen-like atoms with every nucleus, but with their respective muonic orbits about 200 times closer to the nucleus than are the corresponding electron or bits. The 
resulting $x$-ray spectrum directly reflects the charge and electric moment distributions in the nucleus.

Figure 25 is a particularly dramatic representation of the sensitivity oi muonic-atom $x$-ray measurements to changes in the size of the nucleus as one goes from one neutron shell to another. ${ }^{19}$ Quadrupole and hexadecapole moments of nuclei have a'so been determined with extremely good precision by means of muonic-atom $K, L$, and $M$ x-ray measurements. ${ }^{20}$

In addition to information about nuclear charge and magnetic moments, muonic $x$ ray spectra have advanced our knowledge of the extent to which the muon obeys quantum electrodynamics, and have offered the opportunity to look for new types of interartions and to test $\mu-\varepsilon$ universality.

Conservation Laws and Weak-Interaction Theory. The muon is being used at LAMPF, and elsewhere, to test the standard model of electroweak interactions, including the empirical lepton-number conservation laws.

Herbert Anderson and his group have built a spartan but powerful time-projection chamber. They have undertaken the fuimidable task of measuring, with very high accuracy, all the muon-decay parameters, but especialiy $\rho$, predicted by the weak-interaction theory. They aim to analyze 10 -million events to substantialiy improve our knowledge of the parameters of muon decay, which are basic to the theory. TRIUMF measurements have already set a very high standard in this area.

An equally ambitious program involves a Los Alamos/Stanford collaboration, which has developed a very large solid-angle detector (Fig. 26) to search for rare decay modes of the muon-namely, $\mu^{+} \rightarrow e^{+} \gamma, \mu^{+} \rightarrow 3 e$, and $\mu^{+} \rightarrow e^{+} \gamma \gamma$. This group has pushed the first reaction ${ }^{21}$ to its most precise limit, a branching ratio of $\sim 10^{-10}$, and is now contemplating lowering this limit by one to two orders of magnitude. The above decays are not forbidden by present-day formulations of unified theories, and a determination of the probability for lepton-number noncunservation, or even a very small limit on that process, would constitute a substantial advancc. Lepton number violation would imply many chings-finite neutrino mass, heavy lepions, and flavor-changing neut .1 Higgs and neutral horizontal gauge bosons.

Charge Conjug: ion invariance and Conserved Vector Current. A number of LA MPF experiments have been devoted to tests of charge-conjugation invariance and the conserved-vector-current (CVC) hypothesis. The former was tested by searching for the decay $\pi^{0} \rightarrow 3 \gamma$, which would signal C-invariance violation. ${ }^{22}$ It was not found. A new experiment, low under way, will lower the observational threshold by two orders of magnitude. The test applied for CVC was the measurement of the probability for beta decay of the pion-that is, $\pi^{ \pm} \ldots, \pi^{0}+2^{2}+\nu$. CVC was not violated. ${ }^{23}$ The accuracy of this measur^ment was 2.6 times better than that of the best previous determination.

Neutrino Experiments. The beam stop at LAMPF provides an electron-neutrino flux of $\sim 4 \times 10^{7} \nu / \mathrm{cm}^{2} \mathrm{~s}$. Bucause $\pi^{--}$are mostly issorbed, neutrinos are mainly produced from the $\pi^{+}$decay chain. The spectrum includes $30-\mathrm{MeV} \nu_{\mu}$ from $\pi^{+}$decay and 0 - to 53-MeV $\nu_{e}$ and $\bar{\nu}_{1}$ from $\mu^{+}$decay. LAMPF provides a clean source of $\nu_{e}$, as $\nu_{\mu}$ and $\bar{\nu}_{\mu}$ are below threshold for $\mu$ produrtion. 
An experiment has alceady been performed to determine the extent to which leptcnnumber conservation lawy might be multiplicative rather than additive (muon number and electron number separately conserved). ${ }^{24}$ Figure 27 illustrates the principle involved. A 6-ton Cerenkov detector filled with $\mathrm{H}_{3} \mathrm{O}$ was used to look for $\bar{\nu}_{e} p \rightarrow e^{+} n$ and, when filled with $\mathrm{D}_{2} \mathrm{O}$, to measure the reaction $\nu_{e} D \rightarrow$ pe $p$. The first reaction was not observed, the second was. Three infortant results were obtained:

$$
\begin{gathered}
\frac{\mu^{+} \rightarrow \bar{\nu}_{e}}{\mu^{+} \rightarrow \nu_{e}}<0.09 ; \\
\nu_{e} D \rightarrow p e^{-} p=5 \times 10^{-41} \mathrm{~cm}^{2} ;
\end{gathered}
$$

and

$$
\text { so } \bar{\nu}_{\mu} \text { to } \bar{\nu}_{\varepsilon} \text { oscillation. }
$$

$A$ current and much more ambitious neutrino experiment has recently observed the first $\nu_{e}-e$ elastic-scattering events ${ }^{35}$ Just as muonium is an ideal system for studying QED, so $\nu_{e}-e$ scattering is an ideal system for studying the weak interaction in its pure state. The present experiment, involving a University of California, Irvine/Los Alamos rollaboration led by Herbert Chen and Robert Burman, will provide a purely leptonic determination of $\theta_{w}$, the Weinberg angle, which is a crucial parameter in the standard electroweak model.

In the electroweak theory of Glashow, Weinberg, and Salam, a weak neutral-current interaction arises from $7^{0}$ exchange. Its strength is determined by $\sin ^{2} \theta_{w}$, where $\theta_{w}$ is determined by the masses of the $W$ and $Z^{0}$. The $\nu_{e}-e$ cross section is uniquely sensitive to the interference, both in sign and magnitude, between charged- and neutral-current diagrams as follows:

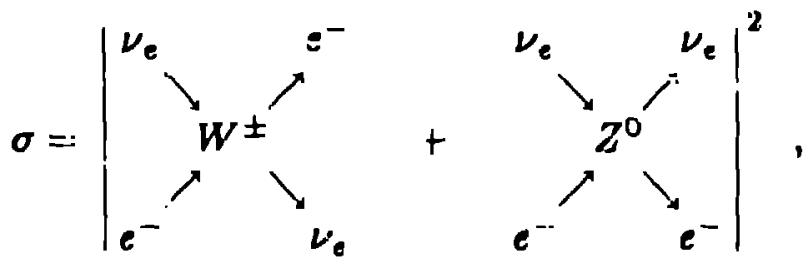

$$
\begin{aligned}
& \sigma=\left\{\left[1+\frac{1}{2}\left(g_{V}+g_{A}\right)\right]^{2}+\frac{1}{3}\left[\frac{1}{2}\left(g_{V}-g_{A}\right)\right]^{2}\right\} ;
\end{aligned}
$$

$$
\text { Standard Model : } \begin{aligned}
g_{V} & =-\frac{1}{2}+2 \sin ^{2} \theta_{U}, \\
g_{A} & =-\frac{1}{2} .
\end{aligned}
$$

'This implies destructive interference between charged and neutral weak currents, with

$$
\sigma_{w e}\left(\nu_{\varepsilon} e^{-}\right) \cong 60 \% \sigma_{V-A}\left(\nu_{e} e^{-}\right)
$$


The prediction was 2 events/day in a 13-ton detector.

In addition tis neutrino-electron scattering, the experimental goals include

$$
\begin{array}{rlrl}
\bar{\nu}_{\mu} & \rightarrow \bar{\nu}_{e} & \text { (neutrino oscillations or } \left.\mu^{+} \rightarrow e^{+} \bar{\nu}_{e} \nu_{\mu}\right), \\
\sigma\left(\nu_{e}{ }^{12} \mathrm{C}\right. & \left.\rightarrow e^{-12} \mathrm{~N}\right), & & \\
\nu_{\mu} & \rightarrow \gamma X & & \text { (neutrino decay) },
\end{array}
$$

and

$$
a_{0} \rightarrow \gamma \gamma \quad \text { (axion production) . }
$$

The detector is surrounded by 3500 tons of shielding material. It contains 9 tons of plastic scintillator and 4 tons of polypropylene flash chambers and measures scattered electrons. It is the presence of hydrogen that permits a sensitive search of one neutrino oscillation channel-namely, $\bar{\nu}_{\mu} \rightarrow \bar{\nu}_{e}$-the reaction being $\bar{\nu}_{e} p \rightarrow e^{+} n$. For maximum mixing and $\Delta m^{2}>0.3 \mathrm{eV}^{2}$, the rate would be $\sim 100$ events/day with a cosmic-ray background of s.t most half that. About $70 \nu_{e}-e^{-}$elastic-scattering events have been identified thus far.

Figure 28 summarizes some particle physics results and demonstrates how LAMPF is being used to unite particle and nuclear physics.

\section{Applications}

The practical applications of LAiVPF-related technology and of LAMFF beams have been many and varied. I mention a few.

LAMPF was the first large accelerator facility designed for complete computer control. ${ }^{26}$ Our experience has influenced virtually every large facility built since.

The structure ${ }^{27}$ we developed to accelerate protons beyond $100 \mathrm{MeV}$ represented a dramatic advance and has since beex: adopted as the accelerator element in all U.S.manufactured megavoltage $x$-ray therapy machines and in others as well. The gross income from sales of these machines now excesds tive total cost of LAMP:

LAMPF rf technology has been adapted to hyperther:nia devices for the treatment of animal tumors ${ }^{28}$ and human eye defects ${ }^{2 \xi 1}$ (Figs. 20 and 30). Experiments are under way to adapt these devices for the treatment of hurnon tumors. If successtul, a simple and relatively inexpensive ruodality for treating accessible tumors will be brought to a large part of the world that does not now have acce:s to advanced treatment technology.

Over a period of about 5 years, the enginering and biomedical foundations were built for the evaluation of negative-pion therapy in the treatment of otherwise untreatuble tumors. ${ }^{30}$ More than 200 patients received such treatment. The program is now being carried forward at SIN in Switzerland and at TRIUMF in Canada.

The LAMPF residual beam is being used to produce a variety of proton-rich radioisotopes to support research programs in more than a dozen leading biomedical centers. Some of these radioisotopes are nearing use in diagnostic and therapeutic medicine. Figure 31 shows the radioisotopes produced during the past 12 months, their uses, and the institutions involved. ${ }^{31}$

Radiopharmaceutical Labeling Research. A program is under way to develop methods for attaching radionuclides to monoclonal antibodies for use in tumor imaging and 
internal radiation therapy. ${ }^{32}$ Monoclonal antibodies and their fragments enable selective targeting of tumors. The labeled antibodies serve as carriers to transport radioisotopes to tumors, thus minimizing total-body radiation dose and radiation damage to normai tissue.

The time required for labeled antibodies to find the turnor antigen and deliver the dose to the tumor is estimated to be 1-3 days. One therefore wouid like radionuclides with a 1- to 3-day half-life. Copper-67 and bromine-77 have suitabie half-lives and nuclear-decay properties for use in tumor imaging and therapy with radiolabeled antibodies. Animal studies are now under way.

Other applications. About $10 \%$ of LAMPF beam is being used for national security studies in the Weapons Neutron Research (WNR) facility (Fig. 32).

This facility has now been augmented with a storage ring, Fig. 33, which will provide short pulses of neutrons of intensity second to no other facility. These pulsed neutrons will be used, with time-of-flight instrumentation, to address not only national security problems but alsc problems in condensed matter physics and biology.

Almost everyone would agree that, at some time in the future, ve will produce fusion energy in a controlled and safe vay. The main thrust, wurld-wide, involves the corfinement of a plasma at temperatures high enough to initiate and sustain füsion. This is an extraordinarily difficult task, which has thus fär eluded our best efforts.

There is an elegant way to produce fusion at room temperature. It involves the use of negative muors to catalyze heavy-hydrogen reactions. Altiough a long way from being feasible as an energy source, $\mu^{-}$-catalyzed $d-T$ fusion has been observed that can produce almost 200 fusions per muon. ${ }^{33}$ The atomic physics is very interesting. A simplified view of the mechanism involved is shown in Fig. 31, prepared by Jim Cohen, Mel Leon, Jin Bradbury, S. Jones, Mike Paciotti, Peter Gram, and Mike Strue actually rather complex, as illustrated in Fig. 35.

Electronuclear breeding devices are already scientifically feasible. The incorpor ation of $\mu$ catalysis will help the economics. It is interesting to observe that if nature had permitted $2000 d-T$ fusions per muon rather than 200 , the problem of devising fusion reactors would be solved.

Finally, a word about the future. We are now planning for the next stage in nuclear science-a kaon factory. Figure 36 shows a plan view of the proposed LAMPF II facility, which will use the $800-\mathrm{MeV}$ linac as an injector. This facility will extend the fronticr of high-energy physics to the region of $50 \mathrm{GeV}$.

\section{ACKNOWLEDGMENTS}

The author is indebted to John C. Allred and Beverly Talley for helpful technical and editorial comments. 


\section{REFERENCES}

1. LAMPF Experiment 645, T. Y. Ling and T. A. Romanowski, Spokesmen.

2. LAMPF Experiments 709,583 , and 790, in "Progress at LAMPF," Los Alamos National Laboratory report LA-10429-PR (April 1985), pp. 28-36.

3. G. W. Hoffmann, L. Ray, M. Barlett, J. McGill, G. S. Adams, G. J. Igo, et al., Physicai Review C 21, 1488 (1980).

4. M. V. Hynes, A. Ficklesimer, P. C. Tandy, and R. M. Thaler, Los Alamos National Laboratory document LA-UR-83-3622 (December 1983); and B. S. Schwarzschild, Physics Today 37, 20 (1984).

5. A. Rahbar, B. Aas, E. Bleszynski, M. Bleszynski, H. Haji-Saeid, C. J. Igo, et al., Physical Review Letters 47, 1811 (1381); and J. R. Shepard, J. A. McNeil, and S. J. Wallace, Physical Reriew Letters 50, 1443 (1983).

6. T. A. Carey, K. W. Jones, J. B. McClelland, J. M. Moss, L. B. Rees, N. Tanaka, and A. D. Bacher, Physical Review Letters 53, 144 (1984).

7. J. J. Aubert, G. Bassompierre, K. H. Becks, C. Best, E. Böhm, X. de Bouard, et al., Phvsics Letłers 123B, 275 (1983).

8. C. H. Llewellyn-Smith, Physics Letters 128B, 107 (1983); and M. Ericson and A. W. Thomas, Physics Letters 124B, 112 (1983).

9. LAMPF Experiment 523, in "Progress at LAMPF," Los Alamos National Laboratory report LA-10429-PR (April 1984), p. 38.

10. LAMPF Experiment 884, in "Progress at LAMPF," Los Alamos National Laboratory report LA-10429-PR (April 1984), p. 50.

11. D. Dehnhard, S. J. Tripp, M. A. Franey, G. S. Kyle, C. L. Morris, R. L. Boudrie, et al., Physical Review Letters 43, 1091. (1979); and N. Auerbach, A. Klein, and E. R. Siciliano, "Isospin Composition of Giant Resonances and Asymmetries in $\pi^{+}$vs $\pi$ - Inelastic Scattering," Los Alamos National I,aboratory document. LA-UR-84-2002 (submitted to Physical Review Letters).

12. H. W. Baer, R. D. Bolton, J. D. Bowman, M. D. Cooper, F. H. Cverna, R. H. Heffner, et al., Nuclear Irstruments \& Methods 180, 445 (1981).

13. J. D. Bowman, H. W. Baer, R. Bolton, M. D. Coope1, F. H. Cverne, N. S. P. King, et al., Physical Review Letters 50, 1195 (1083); and A. Frell, J. Alster, J. Lichtenstadt, M. A. Moinester, J. D. Bowman, M. D. Cooper, et al., Physical Review Letters 52 , 2134 (1984).

14. LAMPF Experiment 852, in "Progress at LAMPF," Los Alamos National Laboratory report LA-10429-PR (April 1984), pp. 74-77.

15. H. C. Bryant, B. D. Dieterle, J. Donahue, H. Sharifian, H. Tootoonchi, D. M. Wolfe, et al., Physical Review Letters 38, 228 (1977); P. A. M. Gram, J. C. Pratt, M. A. YatesWilliams, H. C. Bryant, J. Donaliue, H. Sharifian, and H. Tootoonchi, Physical Review 
Leiters 40, 107 (1978); and J. B. Donahue, P. A. M. Gram, M. V. Hynes, R. W. Hamm, C. A. Frost, H. C. Bryant, et al., Physical Review Letters 48, 1538 (1982).

16. V. W. Hughes, Comments on Nuclear and Particle Physics 12, 259 (1984).

17. A. Badertscher, S. Dhawan, P. O. Egan, V. W. Hughes, D. C. Lu, M. W. Ritter, e al., Physical Review Letters 52, 914 (1984).

18. M. A. Scha, 't, J. S. Frank, C. M. Hoffmann, R. E. Mischke, D. C. Moir, and P. A. Thompson, Physical Review D 23, 639 (1981).

19. E. B. Shera, E. T. Ritter, R. B. Perkins, G. A. Rirker, L. K. Wagner, H. D. Wohlfahrt, et al., Physical Review C 14, 731 (1976); and Y. Tanaka, R. M. Steffen, E. B. Shera, W. Reuter, M. V. Hoehn, and J. D. Zumbro, Physical Review C 30, 350 (1984), and Physical Review C 28, 1897 (1984).

20. Y. Tanaka, R. M. Steffen, E. B. Shera, W. Reuter, M. V. Hoehn, and J. D. Zumbro, Physical Review Letters 51, 1633 (1983); and Y. Tanaka, R. M. Steffen, E. B. Shera. W. Reuter, M. V. Hoehn, and J. D. Zumbro, Physical Review C 29, 1830 (1984).

21. C. M. Hoffman, in the "Proceedings of the 1982 DPF Sumamer Study on Elementary Particle Physics and Future Facilities," Snowmass 1982, R. Drnaldson, R. Gustafson, and F. Paige, Eds., pp. 527-530, conference held at Snowmuss, Colorado, June 28July 16, 1982; R. Bolton, R. Carlini, J. Bowman, M. Cooper, A. Hallin, J. Frank, et al., AIP Conference Proceedings 114, 242-243 (1984); and A. L. Hallin, R. D. Bolton, J. D. Bowman, M. Duong-Van, J. S. Frank, P. A. Heusi, et al., "The Los Alamos Crystal Box Experiment: A Search for $\mu \rightarrow d \gamma, \mu \rightarrow e_{\gamma} \gamma$, and $\mu \rightarrow$ eee," Los Alamos National Laboratory document LA-UR-84-2130 (to be published in the AIP Conference Proceedings on the Intersections Between Particle and Nuclear Physics, Steamboat Springs, Colorado, May 23-30, 1984).

22. V. L. Highland, L. B. Auerbach, N. Haik, W. K. McFarlane, R. J. Macek, J. C. Prati, et al., Physical Review Letters 44, 628 (1980).

23. W. K. McFarlane, L. B. Auerbach, F. C. Gaille, V. L. Highland, E. Jastrzembski, R. J. Macek, et al., Physical Review Letters 51, 249 (1983).

24. S. E. Willis, V. W. Ilughes, P. Némethy, R. L. Burman, D. R. F. Cochran, J. S. Frank, et al., Physical Review Letters 44, 522 (1980); , Physical Review Letters 45, 1370(E) (1980); and P. Némethy, S. E. Willis, V. W. IIughes, R. L. Burman, D. R. F. Cochran, J. S. Frank, et al., Physical Review D 23, 262 (1981).

23. J. S. Frank, T. J. Bowles, R. L. Burman, R. D. Carlini, D. R. F. Cochran, E. Piasetzky, et al., "Observation of $\nu_{e}-e$ Elastic Scattering," presented at the XXII International Conference on High-Energy Physics, Leipzig, Germany, July 19-24, 1984, Los Alamos National Laboratory document LA-UR-84-2325.

26. "Progress at LAMPF," Los Alamos National Lahoratory report LA-10429-PR (1984), p. 224. 
27. D. E. Nagle, E. A. Knapp, and B. C. Knapp, Review of Scientific Instruments 33, 1583 (1957).

28. J. D. Doss and C. W. McCabe, Medical Instrumentation 10, 16-21 (1976); J. D. Doss, "Treatment of Bovine Cancer-Eye (and Other Animal Tumors) with Heat," in Beef Cattle Science Handbook (Agriservices Foundation, Clovis, California, 1980), Vol, 17; and J. D. Doss, Medical Physics 9, 566-573 (1982).

29. J. J. Rowsey, J. R. Gaylor, R. Dahlstrom, and J. D. Doss, Contact \& Intraocular Lens Medical Journal 6, 1-12 (1980); J. D. Doss, R. L. Hutson, J. J. Rowsey, and R. Brown, Archives of Ophthalmology 99, 1261-1265 (1981); and J. J. Rowsey and J. D. Doss, Ophthalmology 88, 755-760 (1981).

30. C. F. von Essen, SIN Jahresbericht 1983, pp. 19-24.

31. "Progress at LAMPF," Los Alamos National Laboratory eport LA-10429-PR (1984), pp. 140-141.

32. "Yrogress at LAMPF," Los Alamos National Laboratory report LA-10429-PR (1984), pp. 145-147.

33. M. Leon, Physical Review Latters 52, 605 (1984); and M. Leon and James S. Coehn, "Ortho- and Paradeuterium Effects in Muon-Catalyzed Fusion," Los Alamos National Laboratory document LA-UR-84-2689 (submitted to Physics Letters A). 


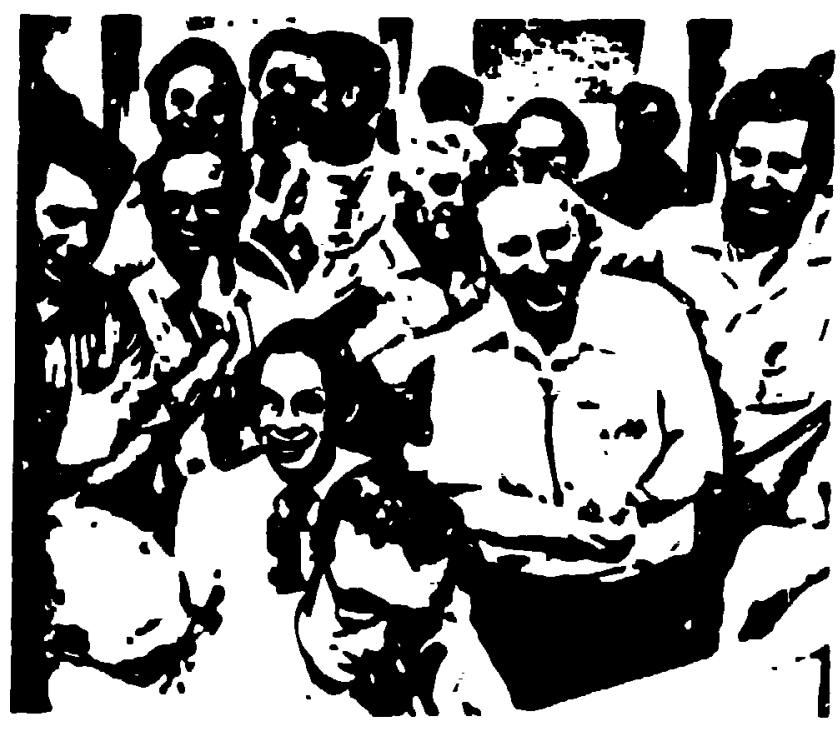

Fis. 1. Jubilation in the control room when LAMPF frat obtained full snergy beam on June 12, 1972.

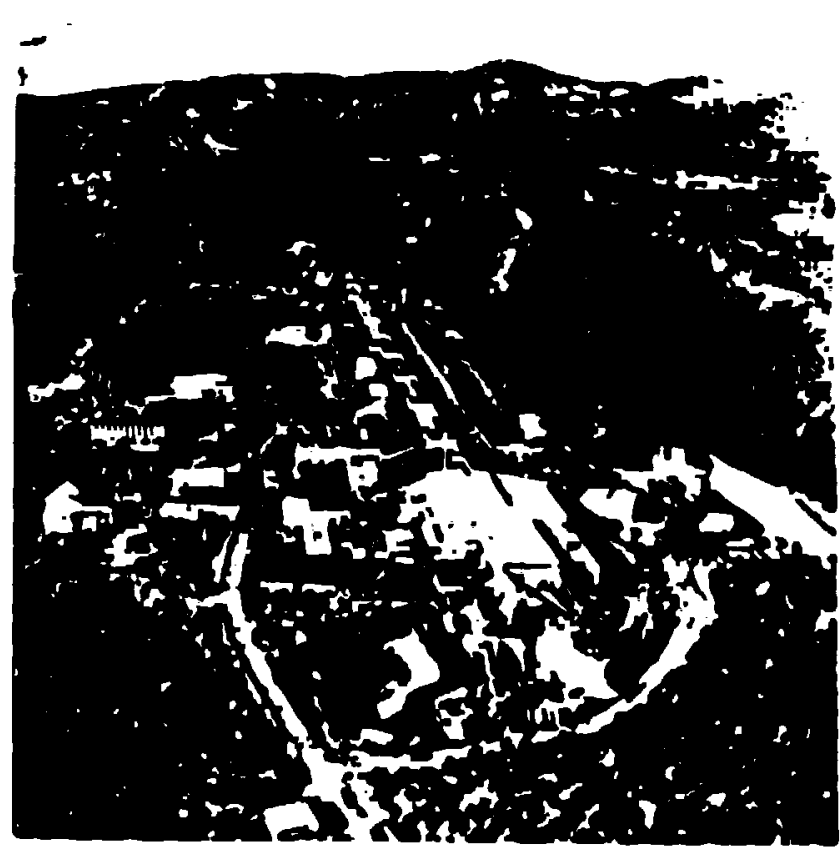

Pic. 2. Atrial view of LAMPF
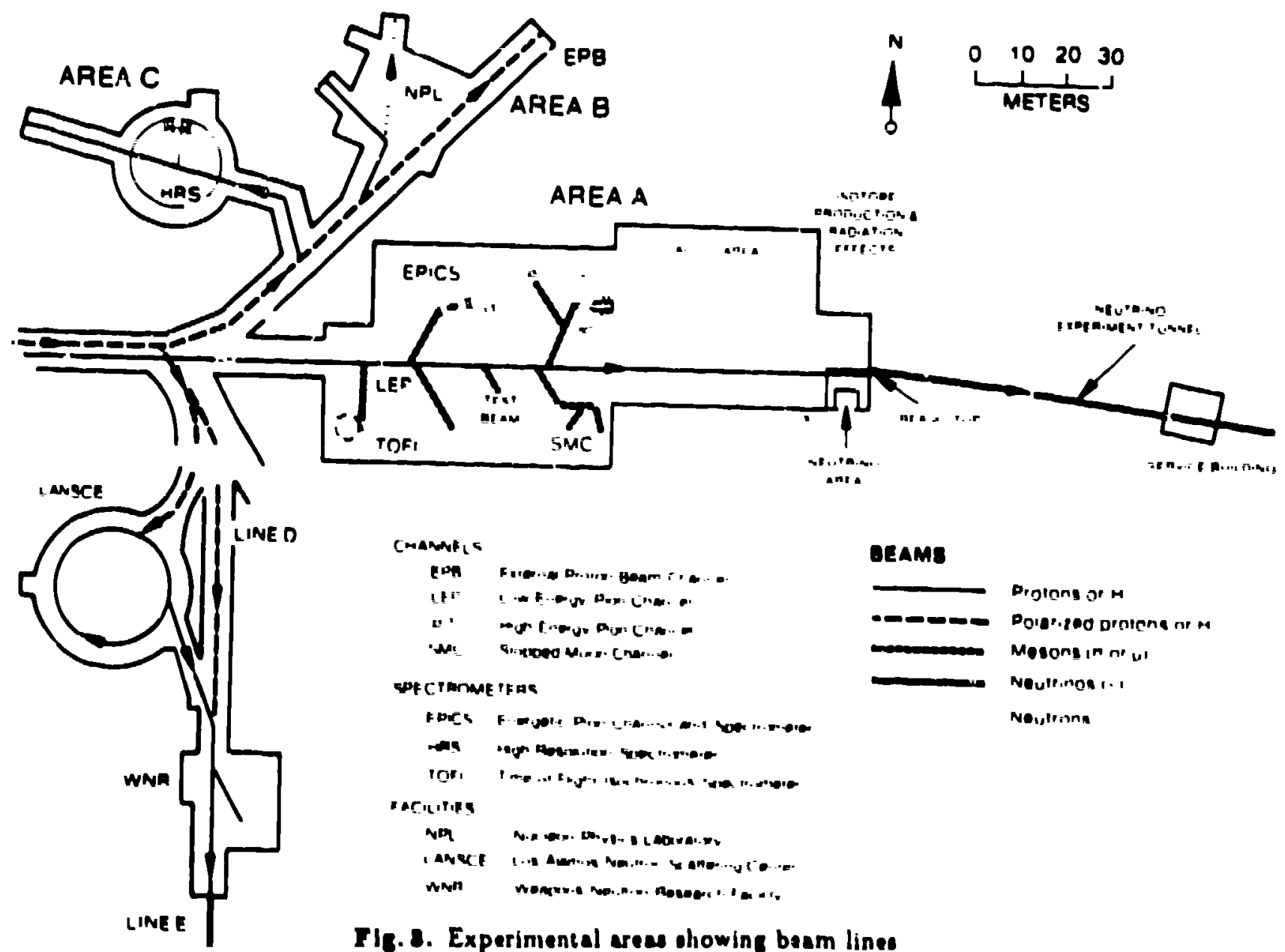

FI. a. Experímental arese diowing beam lines 


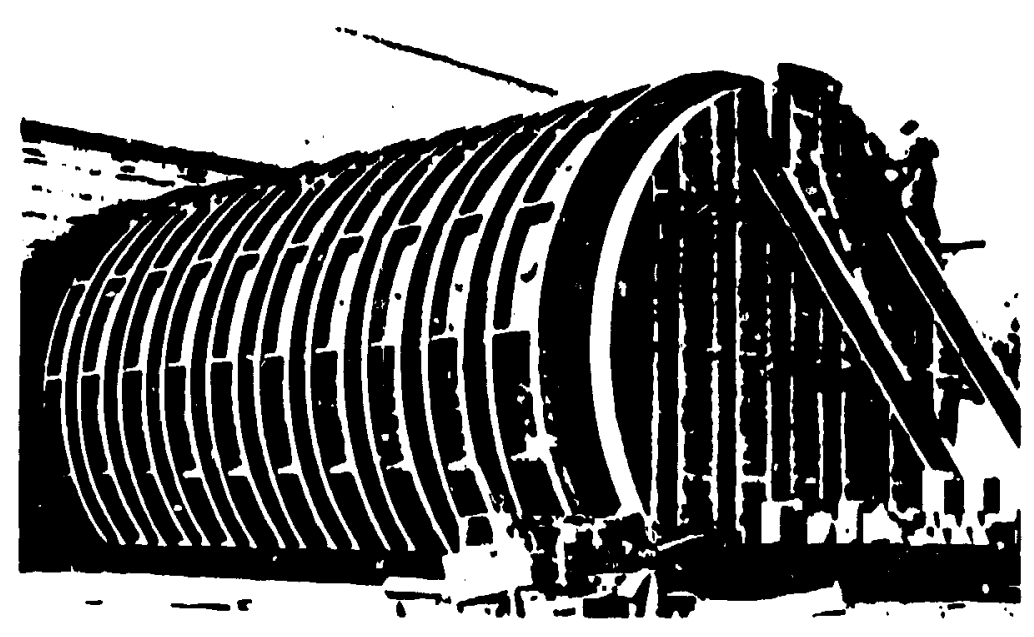

Flg. 8. Organization for governing LAMPF. The 800-member LAMPF Users Group elects a Board of Directors of 7 members, which selects - Technical Advisory Panel and nominates a Program Advisory Commitlee. These three groups (BOD, TAP, and PAC) then interface with LAMPF management on behalf of the lisers. The PAC, appointed by the Director of LAMPF from BOD nominations, advises on experimen. tal priorities.
Fig. 4. Detector housing for neutrino oscillation experiment.

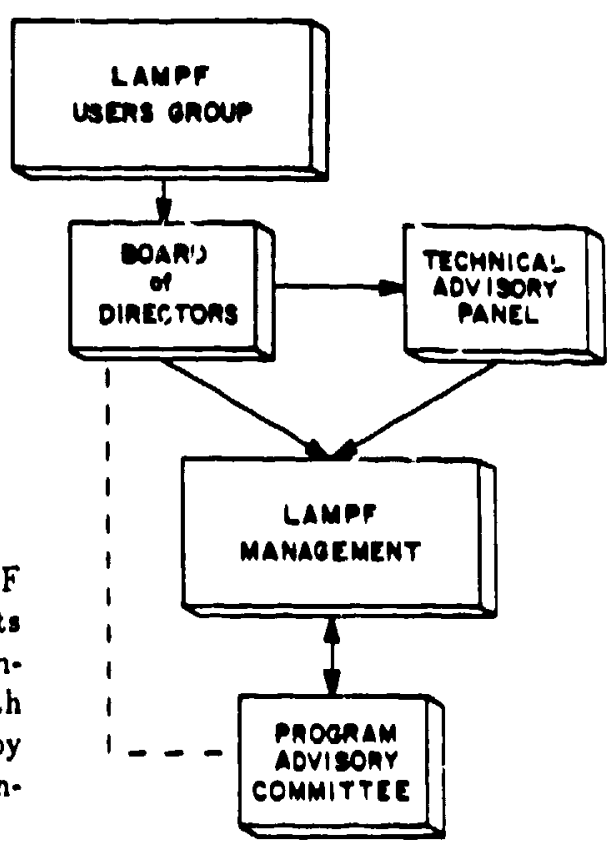

\section{LAMPF : Promises \& Achievements}

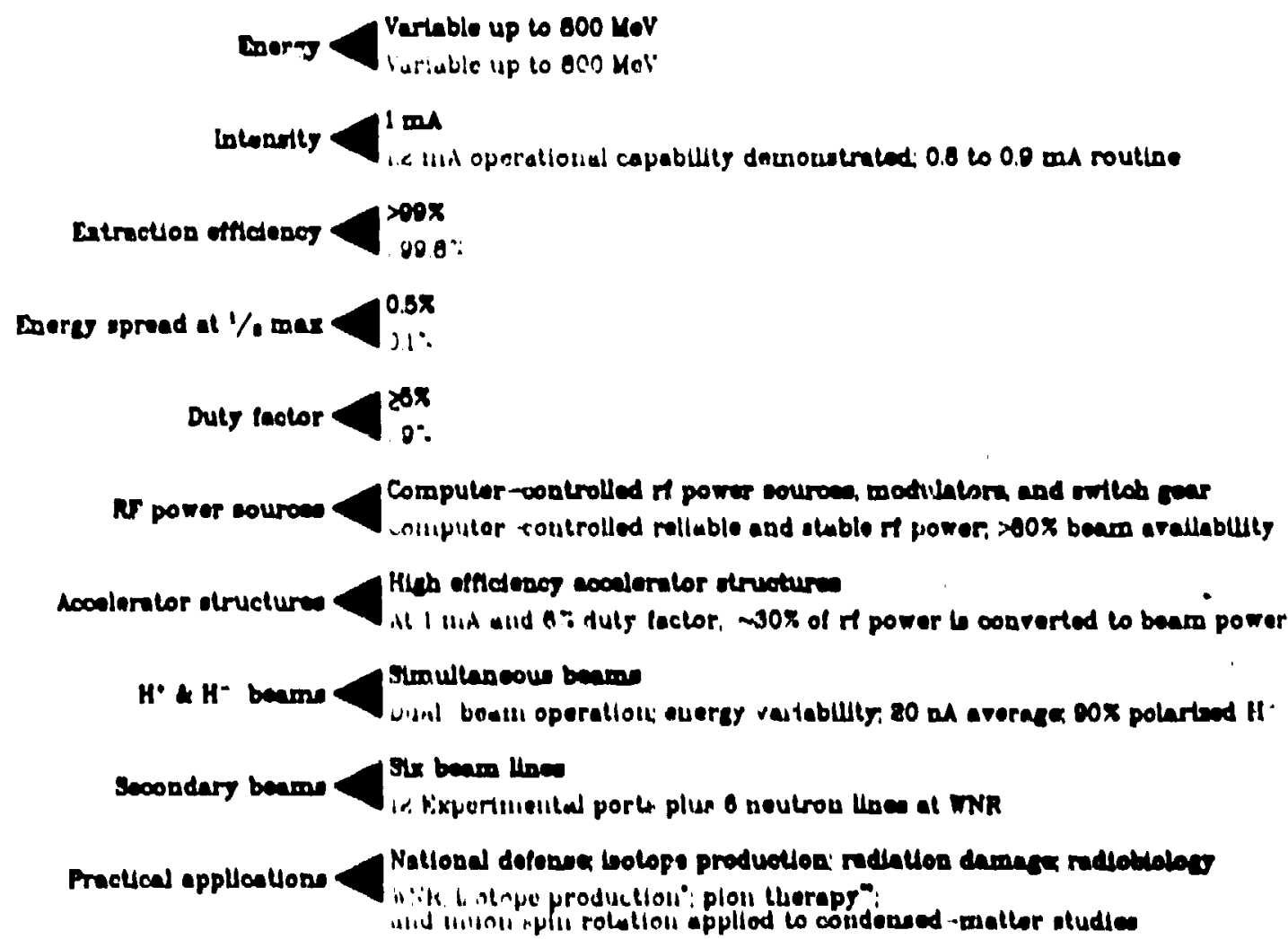

Fis.e. 


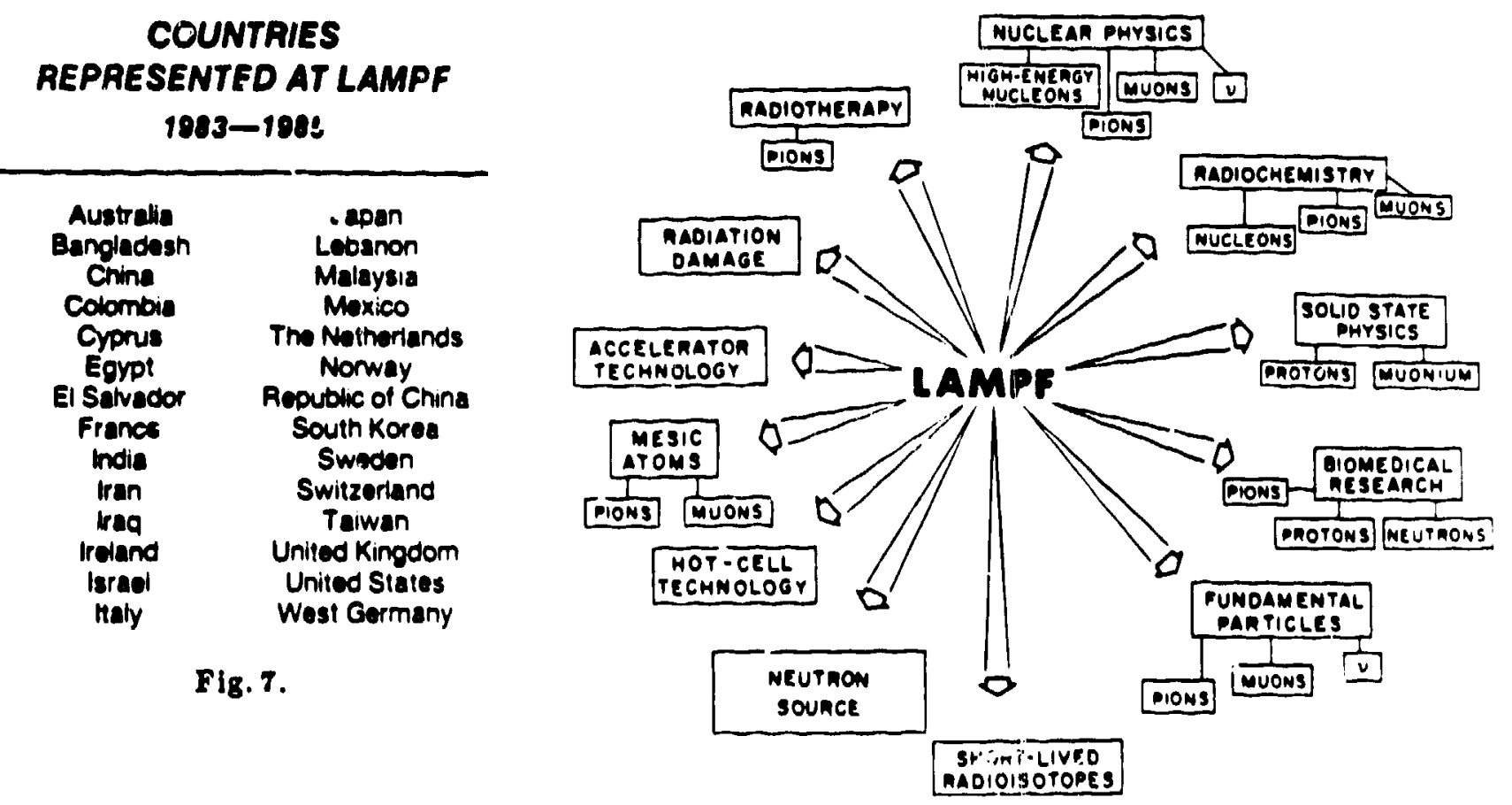

Fig. 8. Diversity of research at LAMPF.

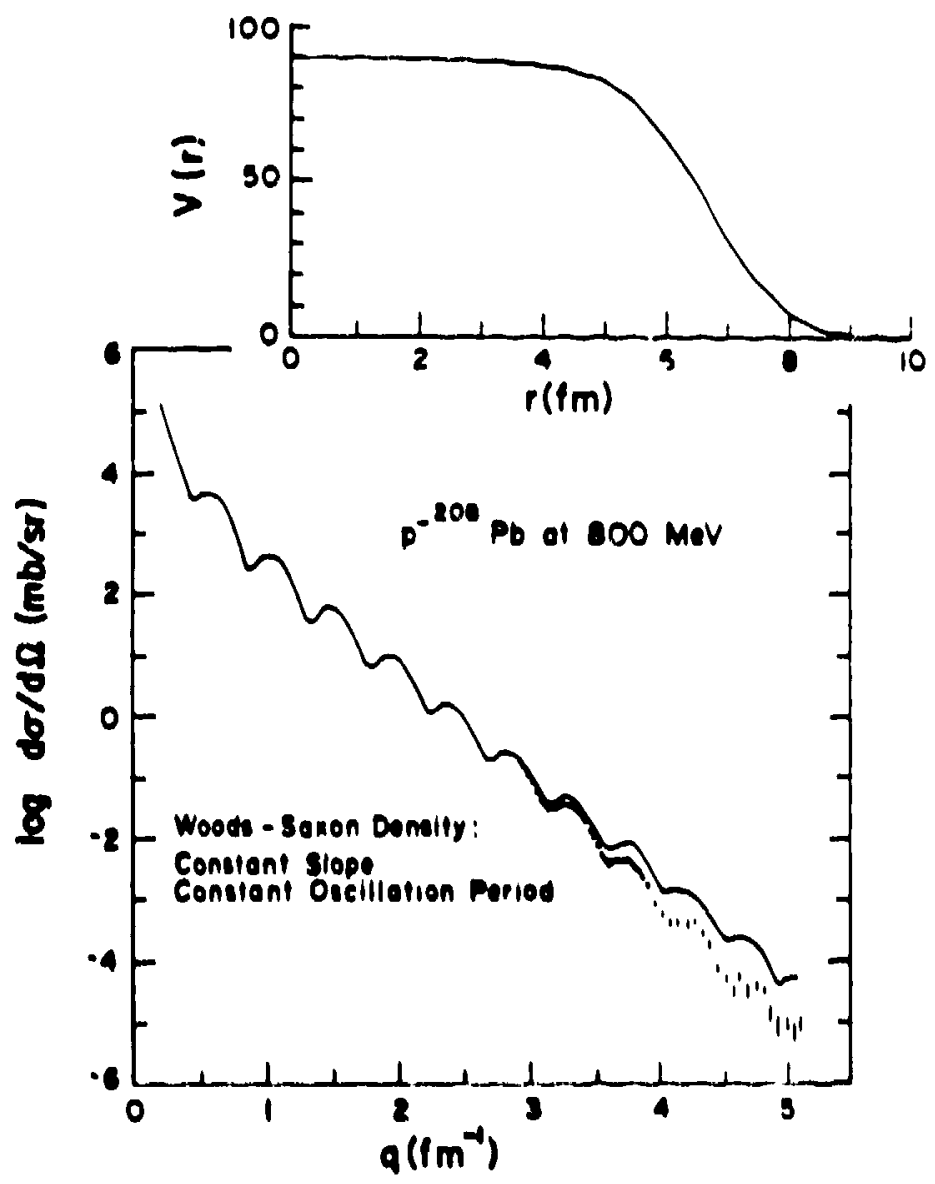

\section{MAJOR RESEAPCH GOALS}

\section{Strong Interactions}

Spin-isospin characteristics

Rolativistic effects

Manifestations of OCD

\section{Electroweak interactions}

Limis of OED

Limils of the Standard Model

Lepton number conservation

Neutrino-electron scattering

Neutrino oscillations

Fig. 9.

Fis. 10. The p-nucleus dave from the High. Recolution Proton Spectrometer showing the diferential erose aection of $\mathrm{p}^{200} \mathrm{~Pb}$ elastic acatterine. The solid curve in a theoret. ical prediction based on the nonrelativiatic Schrödineer equation. 
Pig.11. Firnt mesurement of the opinrotation parameter $Q$ for $500-\mathrm{MeV}$ elasticecattered polaried protons on ${ }^{40} \mathrm{Ce}$ taken with the High-Resolution Proton Spectrometer at LAMPF. The data are compared with full relativintic (solid-line) and nonrelativintic (dahed-line) calculations (see Sbepard et al., Ref. 5).
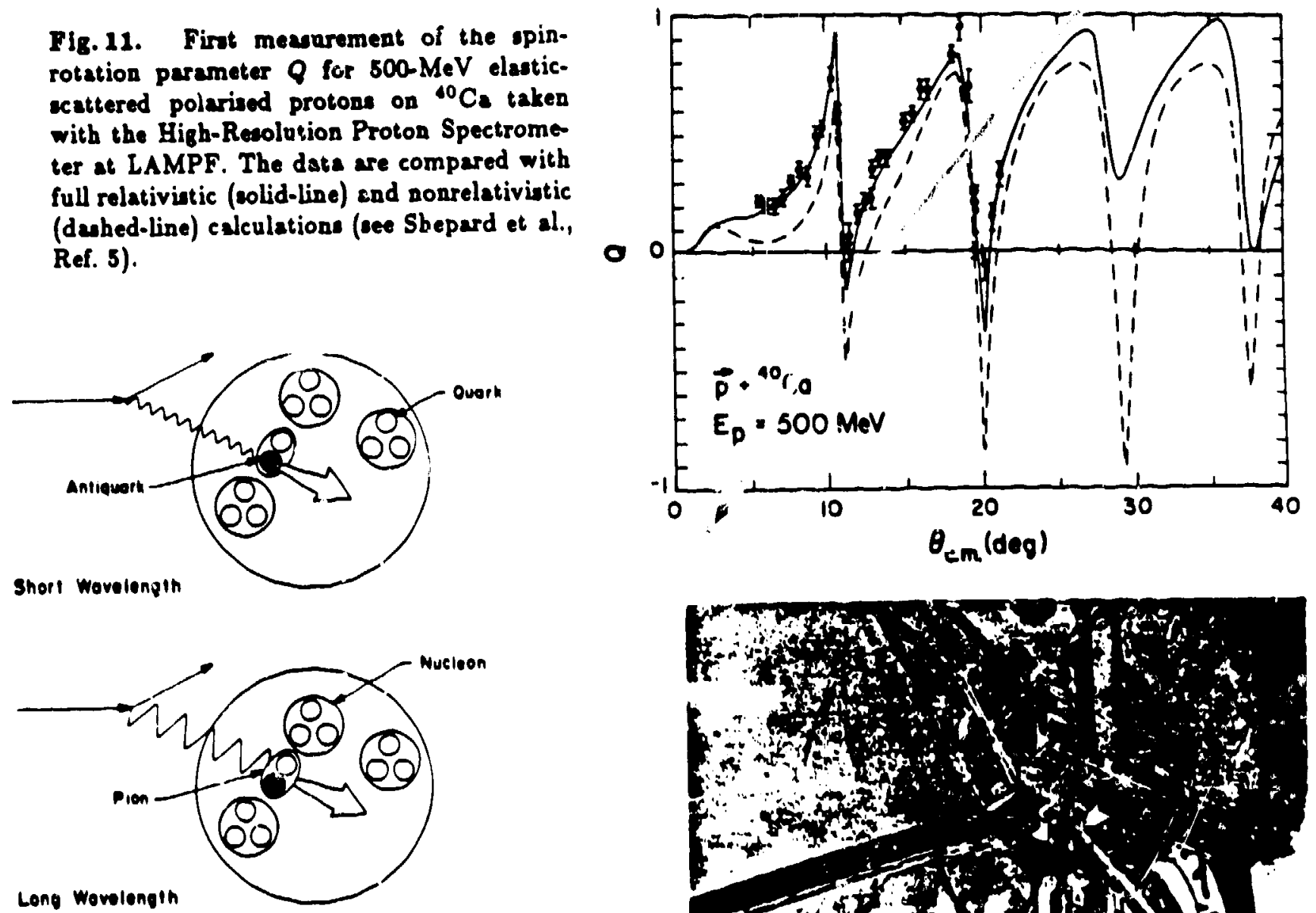

Flg. 13. Schematic representing the nuclear densities probed in the high-energy CERN experiment (top) and medium-energy Los Alemos acattering experiment (bottom). At short wavelengthe the experiniente find quarku and entiquarks ("usnall ohaded eircles). At longer wavelengite cxperimente reveal nucleons (three-quark circles) and pione (querk-entiquark oval).

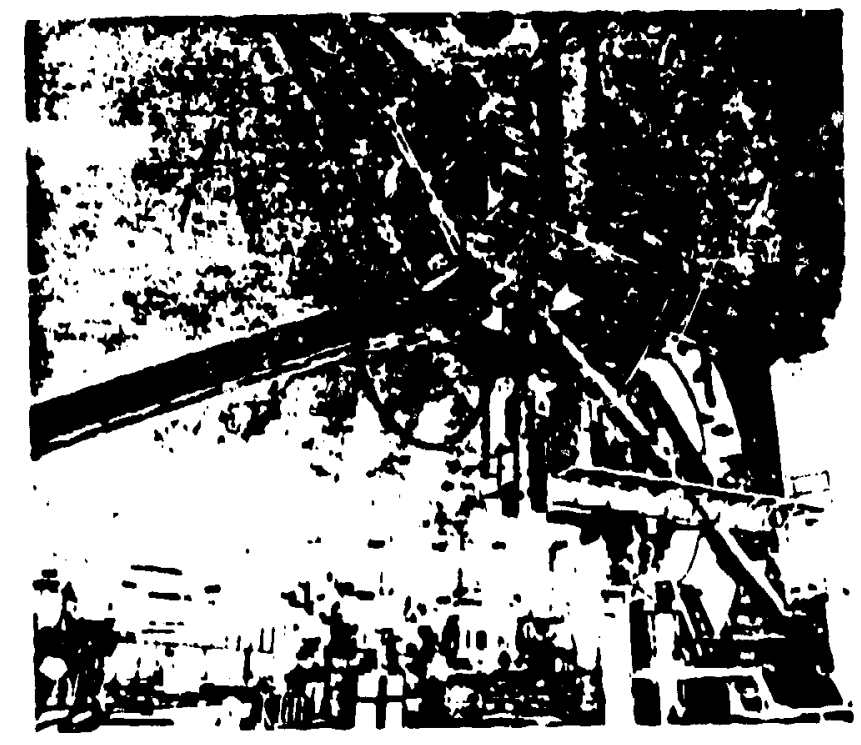

Flg. 12. High-Resolution Proton Spectrometer for 50-keV reselution at $800 \mathrm{MeV}$.

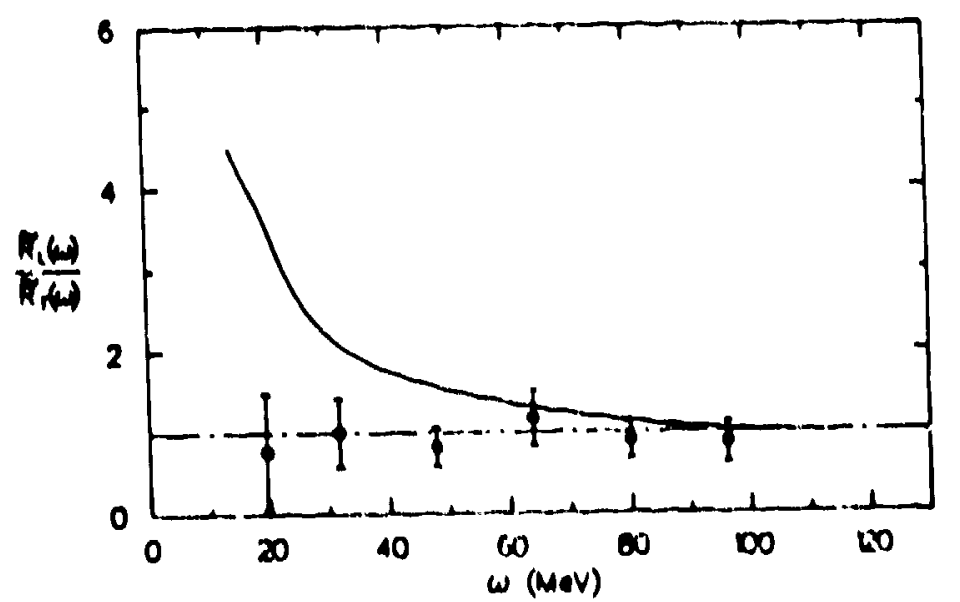

Ig. 14. Pionic ratio date derived from 500 MoV proton eceltering experiments at Los Alemos ( $w$ is the nucleas excitation energy). The curve is the prediction of the pionic en. henceinent model normalised to reproduce the European Muon Collaboration (EMC) effuct. The experimental racio of unity indicales no extre pione in lead. The solid curve is at full nuclear denoity; the dached curve, half nuclear denaity. 


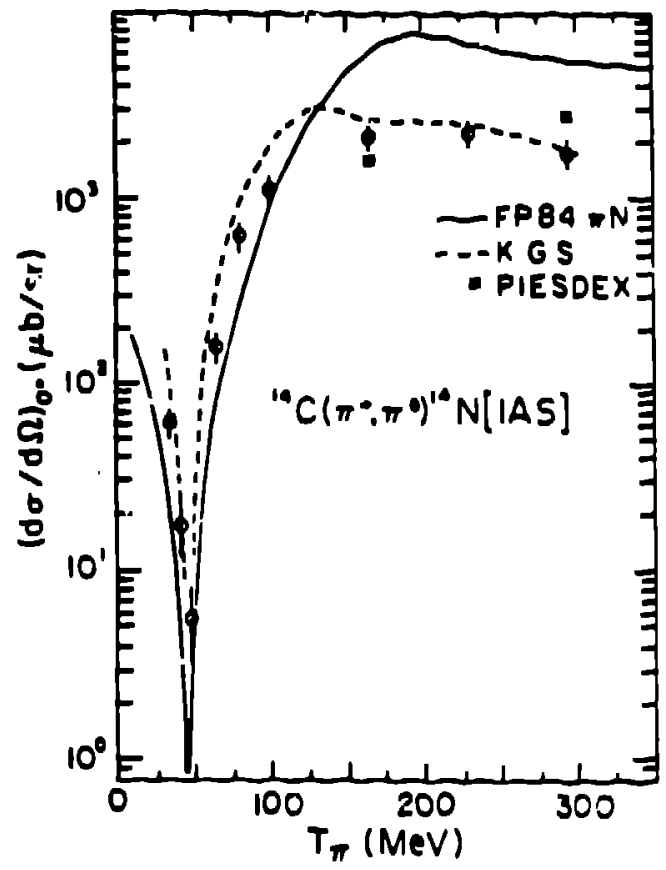

Flg. 15. Zero-degree differential cross sertion $v s$ onergy.

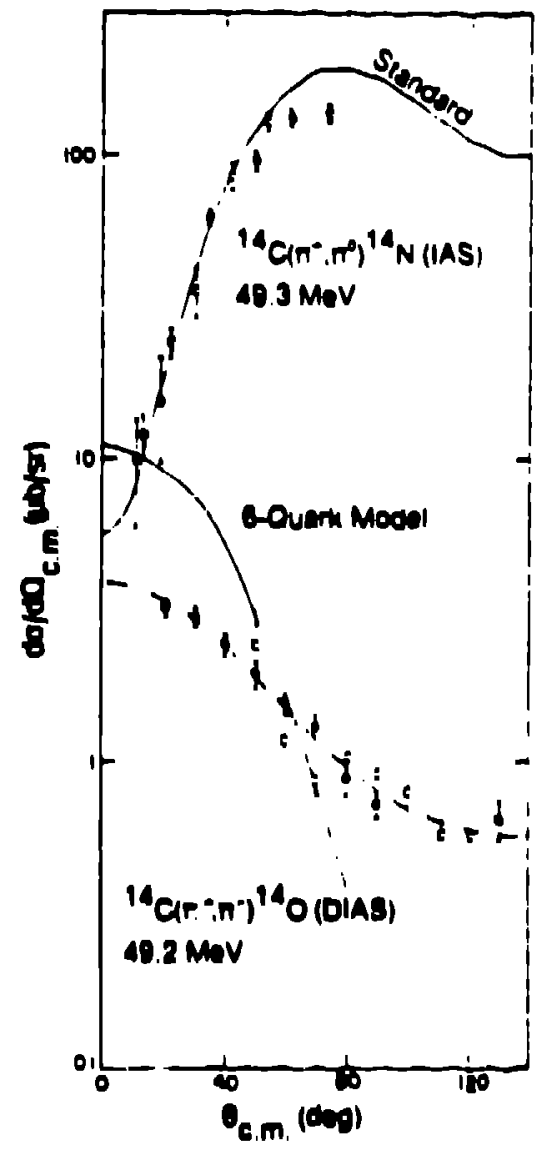

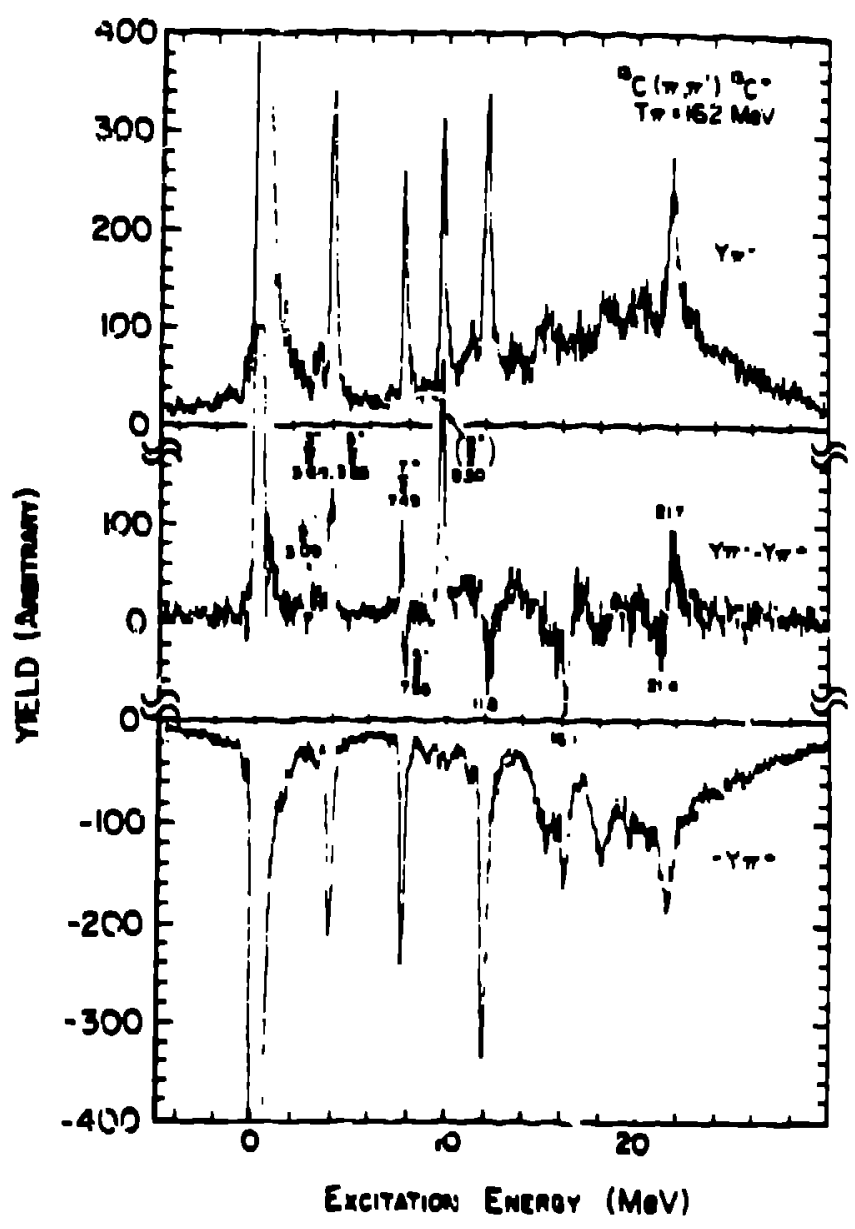

FIg. 17. Inelastic $\pi^{ \pm}$scatcering on ${ }^{13} \mathrm{C}$ vo excitation energy.

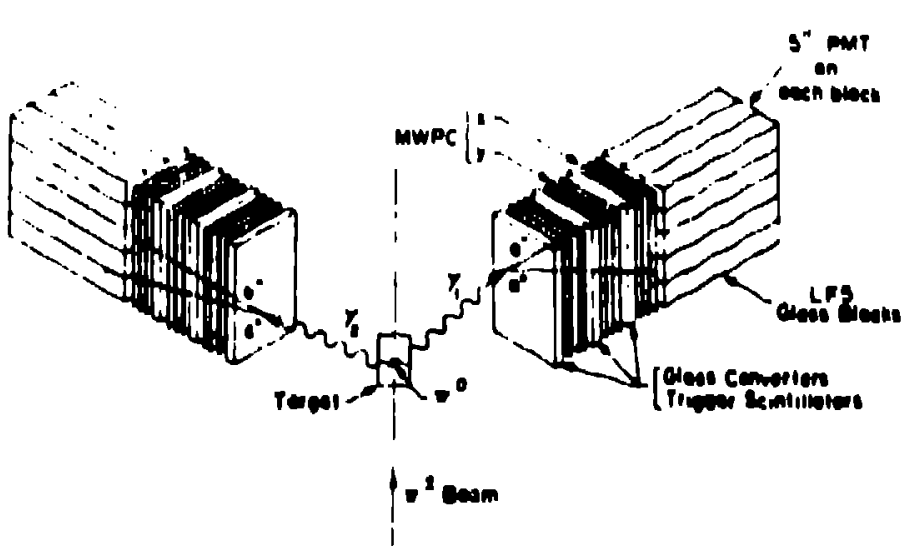

PIg. 18. Components of the $\pi^{\prime \prime}$ epectromater.

FIs. 16. The " $\mathrm{C}\left(\pi^{+}, \pi^{-}\right)^{140}$ crowe cections ve angle and comparion with predlctione of the six-yuark model. 


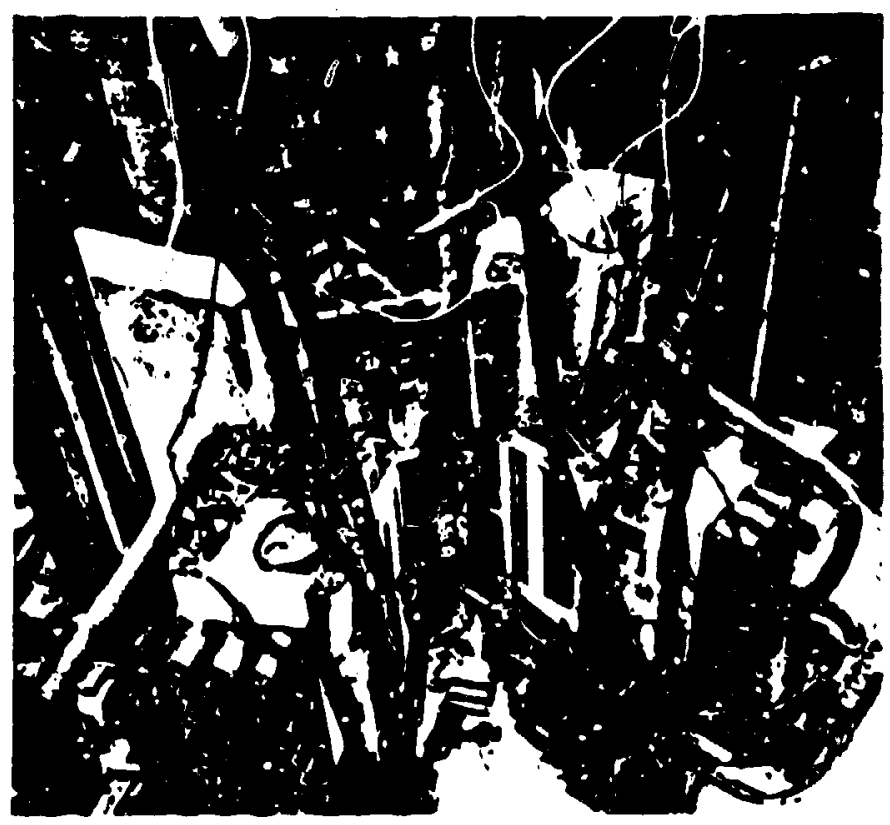

Fig. 19. The $\pi^{0}$ spectrometer installed at the Low-Energy Pion (LEP) chanuel.

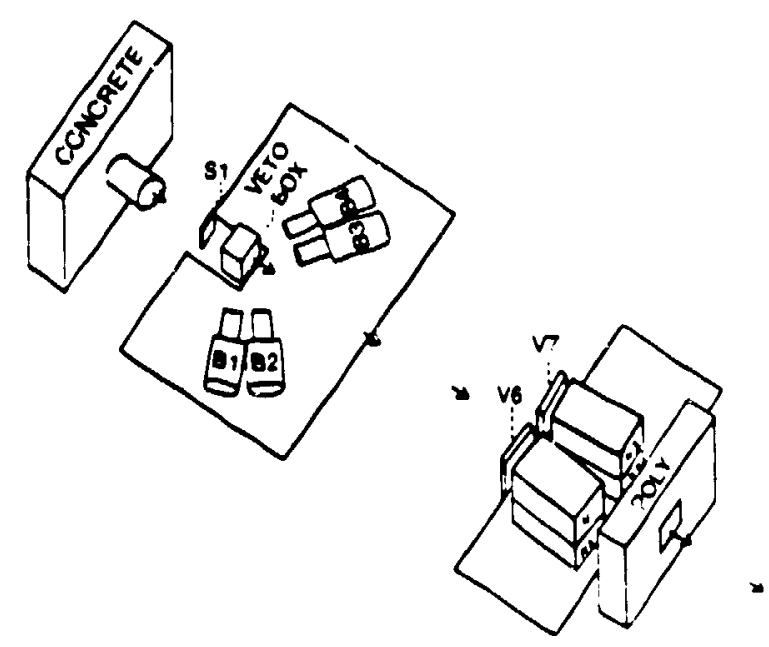

Fig. 21. Schematic of the experimental setup for the test run of $\eta$ production on hydrogen and ${ }^{12} \mathrm{C}$.

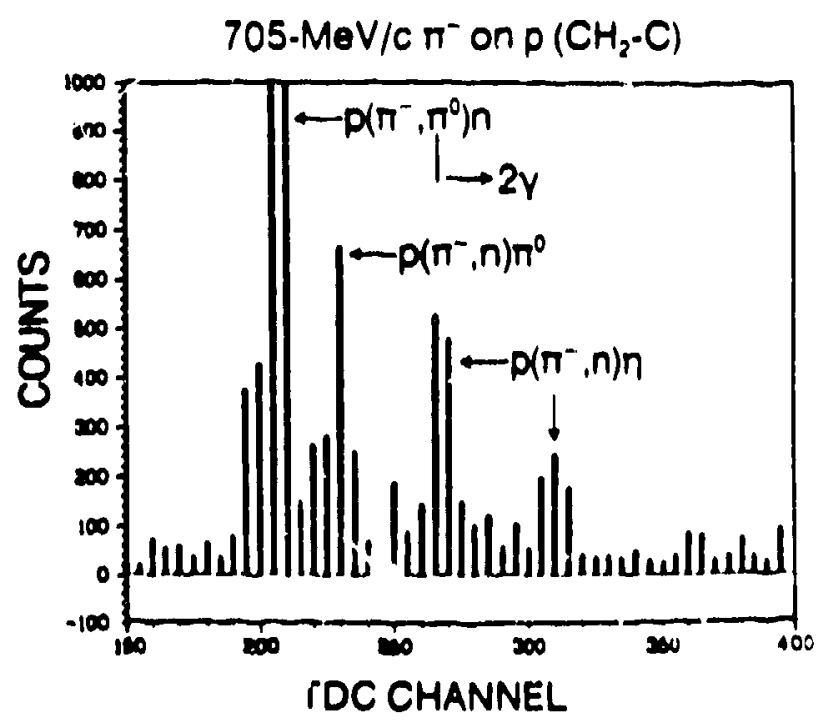

Fig. 22. Neutron time-of-0ight opectrum.

F18. 20. Difierences in the 4.5 and $11.0^{\circ}$ double tifferential crow section for the reaction ${ }^{120} \mathrm{Sn}\left(\pi^{\circ}, \pi^{0}\right)$ at $T_{n-}=163 \mathrm{MeV}, 4$ a function of the kinetic energy of the outgoing $\pi^{\circ}$. The positive-going hump (IVM) is the isovector monopule resonance, which has a maximum at $\theta=0^{\circ}$. The negative going hursp (GDR) is the well-known gient dipole resonence, which has a maximum at $11.0^{\circ}$.

$$
\text { f }
$$




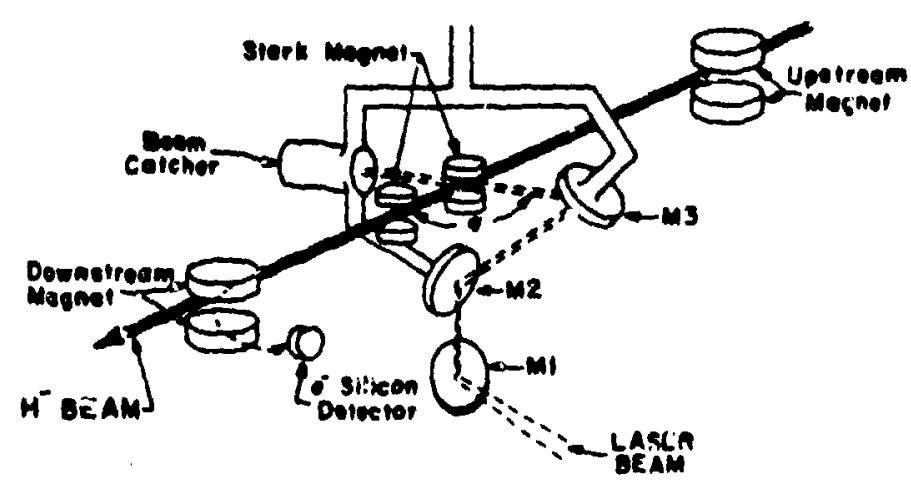

Fig. 23. Atomic physics witin LAMPF: photodetachment of the negative hydrogen ion $\left(\mathrm{H}^{-}\right)$.

Fig. 24. Comparison of photodetachment data with the calculated cross sections of Broad and Reinhardt. Error bars reflect counting statistics only.

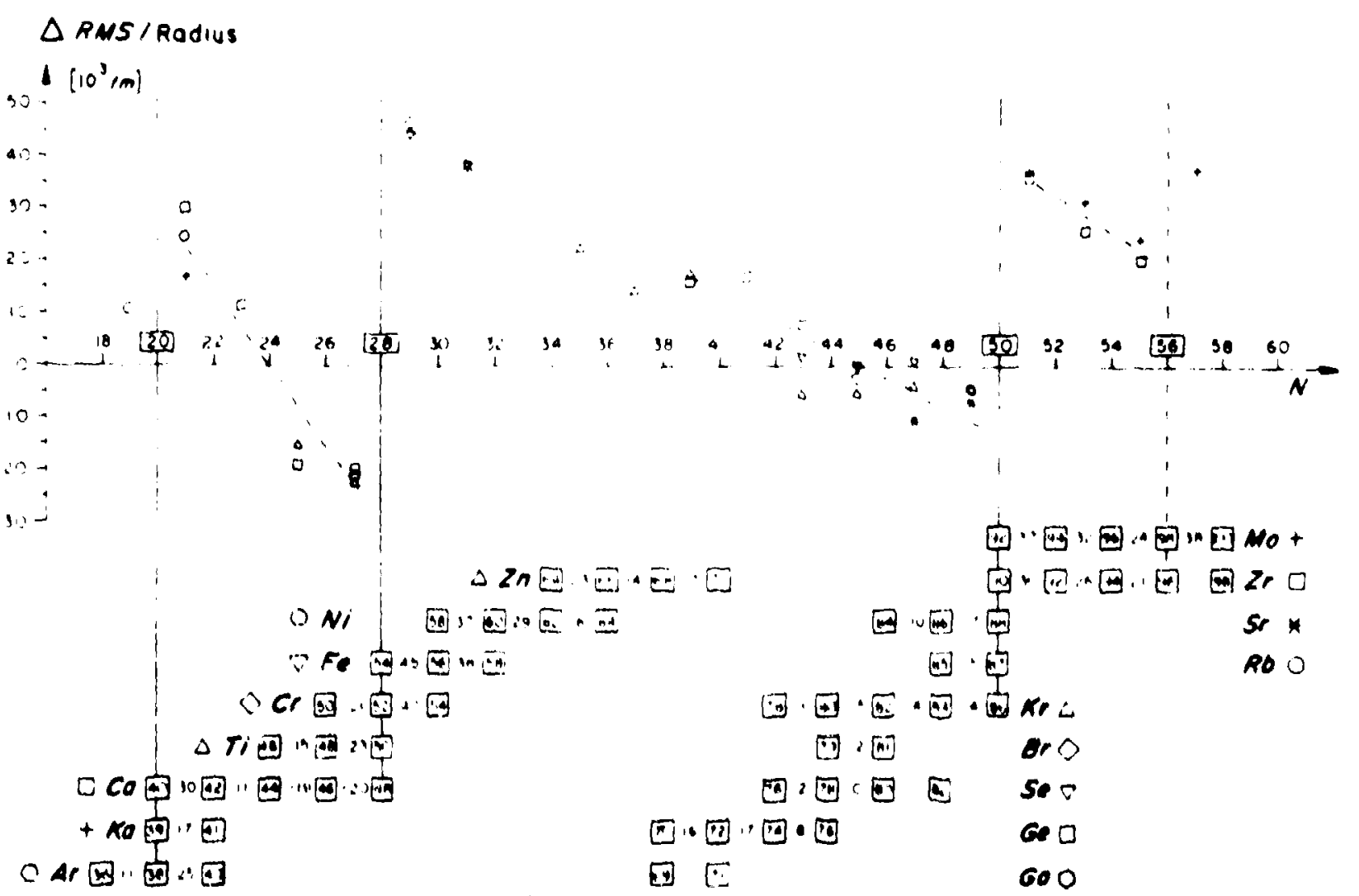

Fig. 28. Differences in nuclear charge radii that occur upon the addition of a poir of neutrons, as rovealed by muonic x-ray masouremente. 


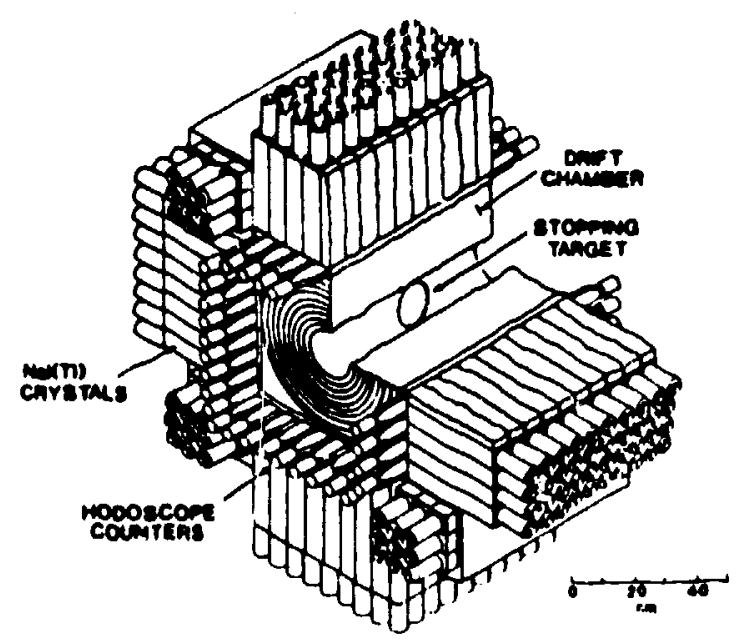

Fig. 26. The Crystal Box detector.

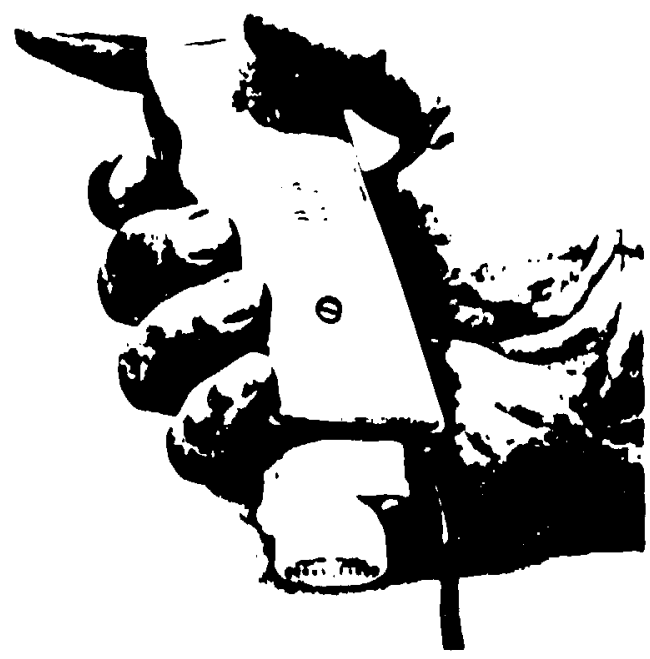

Fig. 29. Hyperthermia device for treating cancer eye in cattle.
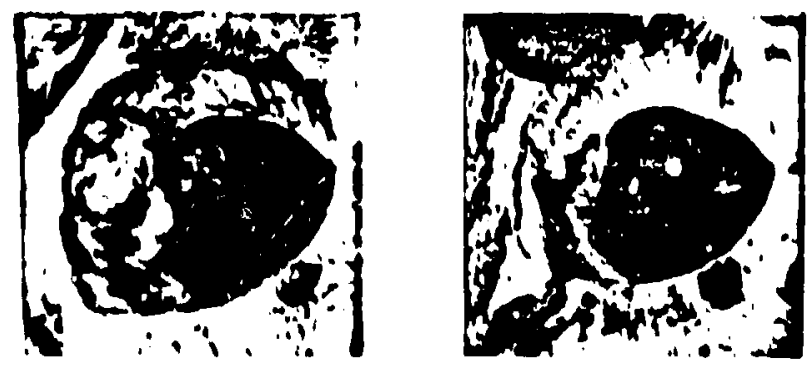

- 2 (HEREFORD) November 17,1976
A Test of Muon Conestration
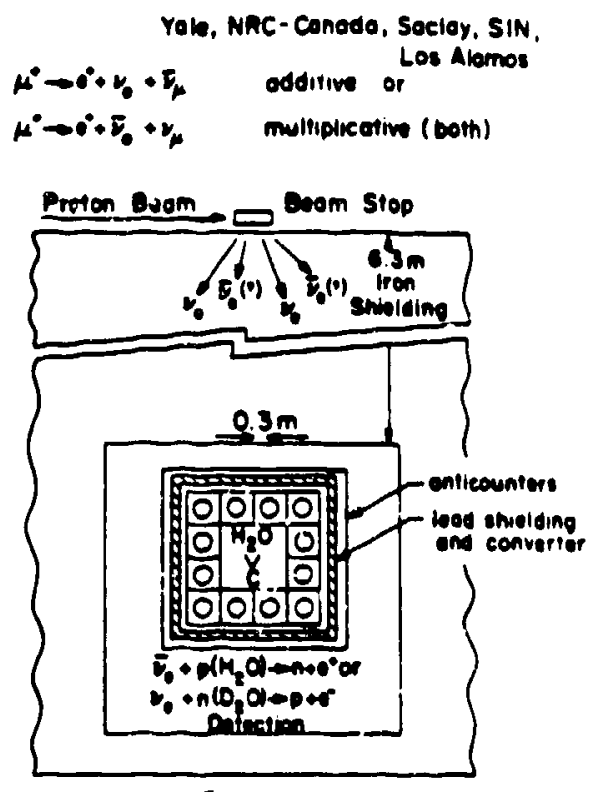

Top Viev

Fig. 27. Schematic of Experiment 31.

\section{LAMPF RESULTS OF INTEREST \\ TO PAATICLE PHYSICS}

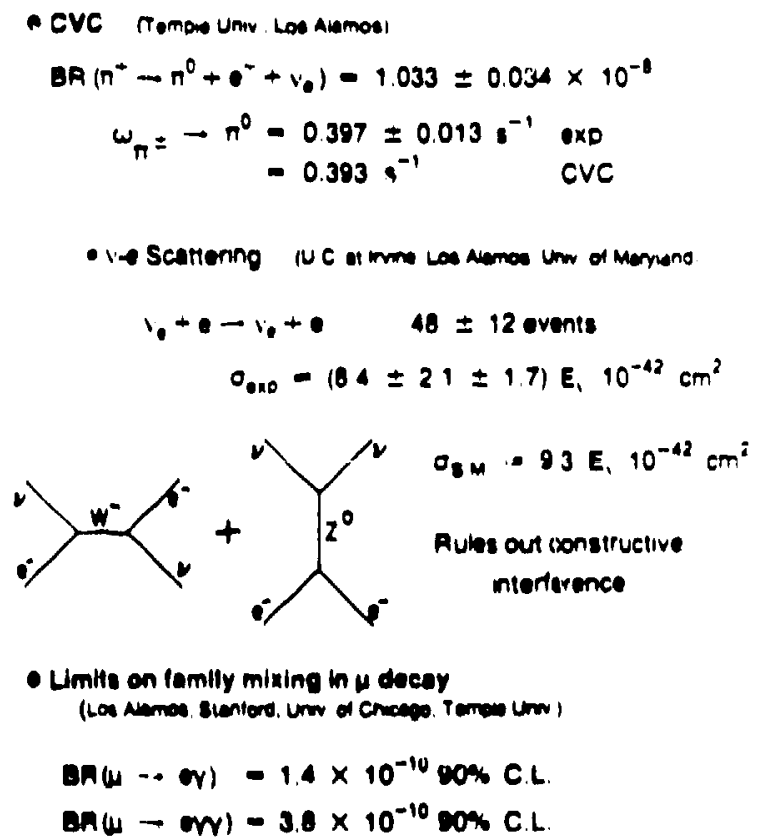

Fig. 28.

Fig. 30. Cancer eve in cattle before and after treatment with the LAMPF hyperthermia device. 


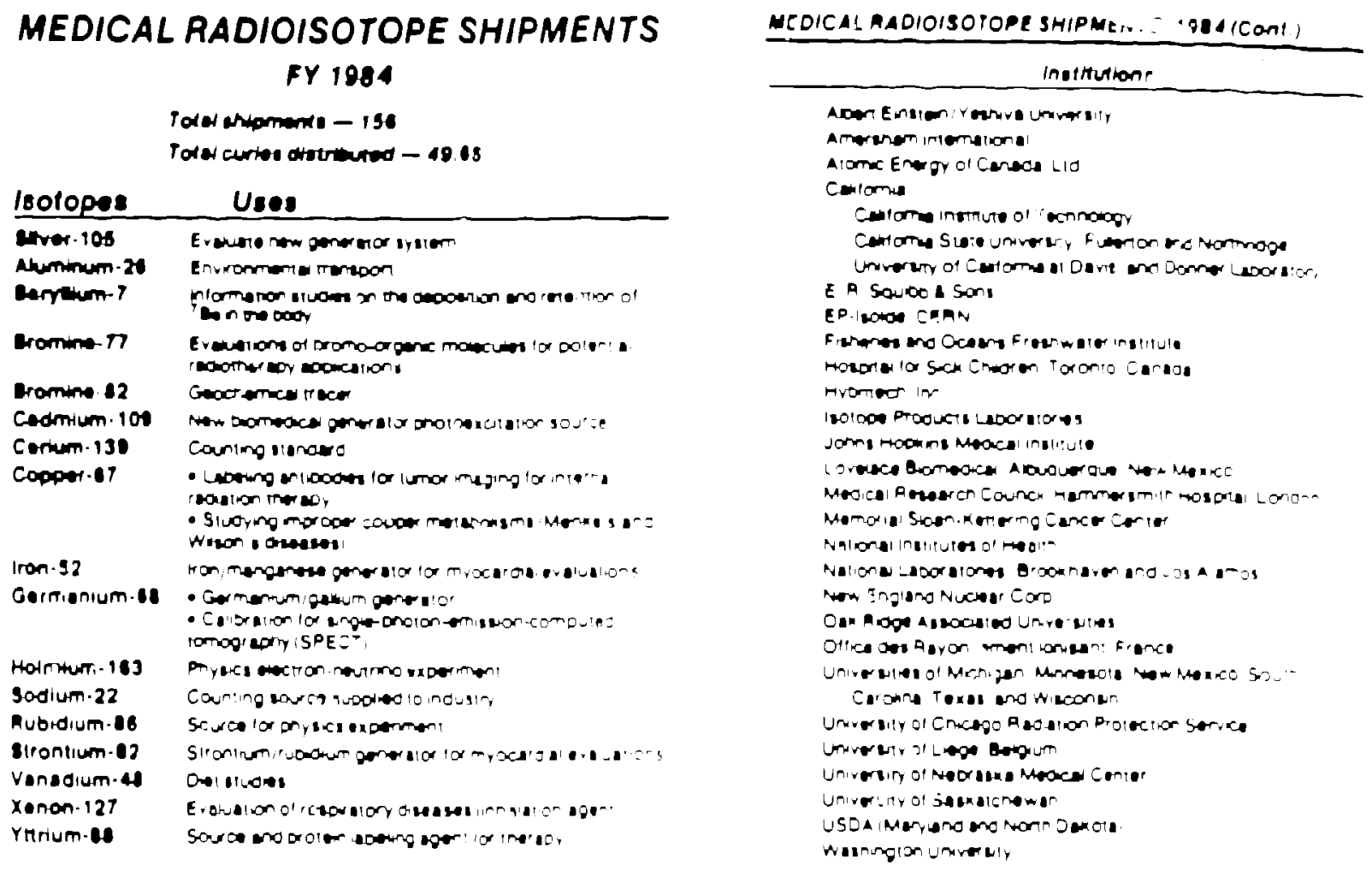

Fig. 31

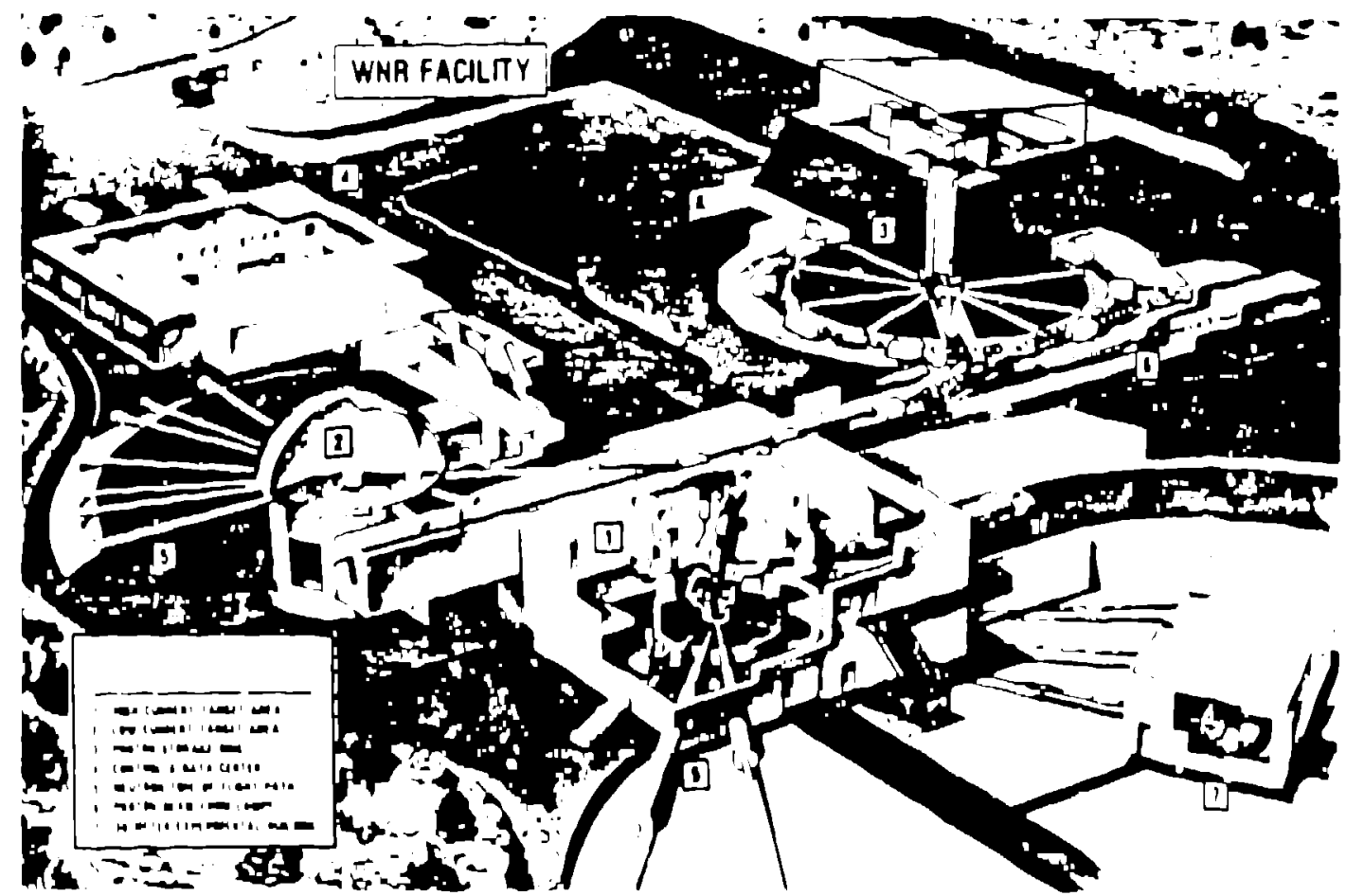

FIg.a2. Weapons Nou iun Reseasch (WNR) facility 

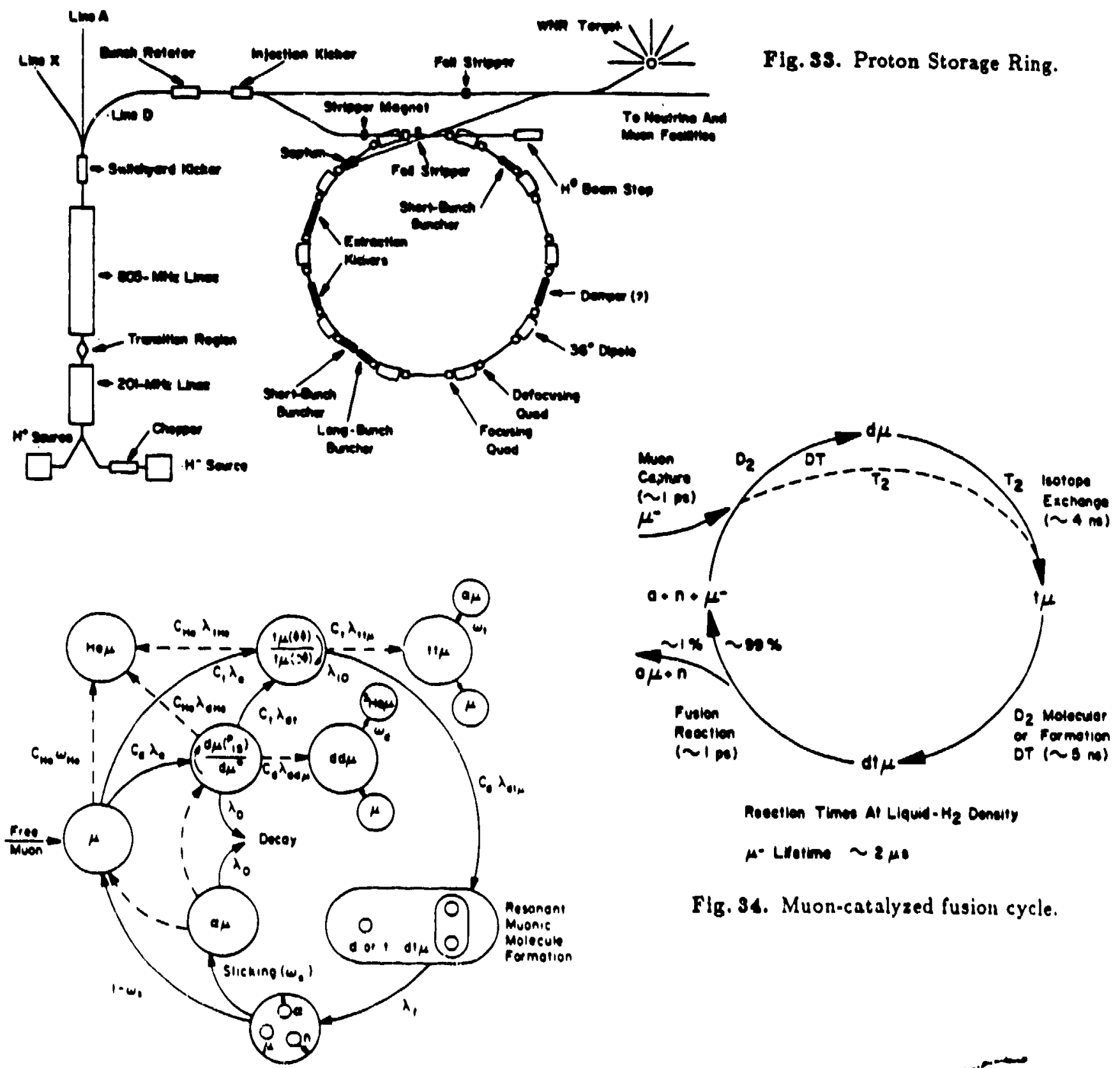

Fig. 3s. Proton Storage Ring.

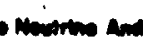

Ten Pcomine

sies

(1) 